\title{
INFINITESIMALS AS AN ISSUE OF NEO-KANTIAN PHILOSOPHY OF SCIENCE
}

\author{
THOMAS MORMANN AND MIKHAIL G. KATZ
}

\begin{abstract}
We seek to elucidate the philosophical context in which one of the most important conceptual transformations of modern mathematics took place, namely the so-called revolution in rigor in infinitesimal calculus and mathematical analysis. Some of the protagonists of the said revolution were Cauchy, Cantor, Dedekind, and Weierstrass. The dominant current of philosophy in Germany at the time was neo-Kantianism. Among its various currents, the Marburg school (Cohen, Natorp, Cassirer, and others) was the one most interested in matters scientific and mathematical. Our main thesis is that Marburg neo-Kantian philosophy formulated a sophisticated position towards the problems raised by the concepts of limits and infinitesimals. The Marburg school neither clung to the traditional approach of logically and metaphysically dubious infinitesimals, nor whiggishly subscribed to the new orthodoxy of the "great triumvirate" of Cantor, Dedekind, and Weierstrass that declared infinitesimals conceptus nongrati in mathematical discourse. Rather, following Cohen's lead, the Marburg philosophers sought to clarify Leibniz's principle of continuity, and to exploit it in making sense of infinitesimals and related concepts.
\end{abstract}

KEYWORDS: Infinitesimals; Marburg neo-Kantianism; principle of continuity; Cantor-Dedekind-Weierstrass; Hermann Cohen; Cassirer; Natorp; Leibniz.

\section{Contents}

1. Introduction 2

2. Neo-Kantian Philosophy of Science and Mathematics 4

2.1. The Transcendental Method 5

2.2. Concepts in Mathematics and Science 7

2.3. Substantive versus Relational Concepts 9

3. Two Guiding Metaphors of neo-Kantian philosophy of science

3.1. Natorp's Knowledge Equation

2000 Mathematics Subject Classification. Primary 01A60, Secondary 03A05, 01A85, 26E35. 
3.2. Cassirer's Convergent Series 15

3.3. Idealisations, completions, and infinitesimals 17

4. Three attempts to make sense of Cohen 19

4.1. The Point of departure: Cohen's Prinzip 20

4.2. Cassirer's Leibniz' System 24

4.3. The Continuity principle 26

4.4. Gawronsky's The Judgment of Reality 32

4.5. Natorp's The Logical Foundations of the Exact Sciences 37

5. From infinitesimals to functional concepts $\quad 42$

Acknowledgments $\quad 47$

References 47

\section{INTRODUCTION}

The traditional historical narrative concerning infinitesimals runs as follows. The idea of infinitesimals has been with us since antiquity. Mathematicians have used one or another variety of infinitesimals or indivisibles without really understanding what they were doing. Eventually, infinitesimals fell into disrepute for logical and philosophical reasons, as enunciated by Berkeley and others.

Despite Berkeley's devastating criticism, mathematicians continued to use them until the 19th century with more or less good intellectual conscience. Finally (according to the traditional narrative) Cauchy, followed by Cantor, Dedekind, and Weierstrass, succeeded in formulating a rigorous foundation for the calculus in terms of the epsilon-delta approach. Thereupon infinitesimals were "officially" expelled from the realm of legitimate mathematics once and for all. Or so it seemed.

This traditional narrative is, however, seriously incomplete. Some 80 years after mathematics had allegedly dismissed "infinitely small magnitudes" and related concepts as pseudo-concepts once and for all, in 1960 the mathematician Abraham Robinson claimed to have saved infinitesmals from the bin of pseudo-concepts. Thereby, an improved version of the traditional narrative goes, Robinson restored the reputation of infinitesimals as legitimate mathematical entities by means of his non-standard analysis. From the perspectives of mathematics and history of mathematics, such a completed version of the traditional narrative is certainly to be considered as an improvement. Nevertheless it still suffers from serious shortcomings. For centuries, the infinitesimal and related concepts were discussed not only by mathematicians and scientists but also by philosophers like Leibniz, Newton, Malebranche, and Berkeley. While the thought of these classic 17th and 18th century 
authors has been extensively studied by historians of philosophy and mathematics, not much is known about the 19th century philosophical context in which the "great revolution in rigor" and the alleged dismissal of infinitesimals took place. This is hardly acceptable. A philosophically satisfying account has to take into consideration the historical fact that the "great revolution in rigor" in mathematical analysis, led by the German mathematicians Cantor, Dedekind, and Weierstrass (henceforth, CDW), took place when philosophy in Germany was dominated by various currents of neo-Kantian philosophy. Some of the neo-Kantian philosophers had a keen interest in science and mathematics. Indeed, the issue of the infinitesimal was vigorously debated in neo-Kantian quarters, as we will discuss in detail in this text.

Our main thesis is that the Marburg neo-Kantians elaborated a philosophically sophisticated approach towards the problems raised by the concepts of limits and infinitesimals. They neither clung to the obsolete traditional approach of logically and metaphysically dubious infinitesimals 1 nor whiggishly subscribed to the new orthodoxy of the "great triumvirate" (Cantor, Dedekind, Weierstrass) that insisted on the elimination of infinitesimals from any respectable mathematical discourse in favor of a new approach based on the epsilontic doctrine. Instead, the Marburg school developed a complex array of sophisticated, albeit not always crystal-clear, positions that sought to make sense of both infinitesimals and limit concepts. With the hindsight enabled by Robinson's non-standard analysis, the Marburg stance seems wiser than that of Russell, Carnap, and Quine who unconditionally accepted the orthodox epsilontic doctrine, along with its simplistic philosophical ramifications stemming from a strawman characterisation of infinitesimals as a pseudo-concept.

The outline of this paper is as follows. In Section 2, we recall the basics of the Marburg school's neo-Kantian philosophy of science and mathematics. In particular, we dwell on some crucial features of the Marburg account that distinguishes it from the Kantian orthodoxy. We also recall the basic ingredients of Cassirer's philosophy of science

\footnotetext{
${ }^{1}$ Actually, there are good reasons to contend that the infinitesimals of the traditional approach were neither logically nor metaphysically dubious, though they were attacked as such by George Berkeley. Sherry 63] dissected Berkeley's criticism (of Leibnizian infinitesimal calculus) into its logical and metaphysical components, and a closer inspection thereof reveals that the Leibnizian system for differential calculus was both more firmly grounded than the Berkeleyan criticism thereof, and free of logical contradictions (Katz \& Sherry 2012 [38]), (Katz \& Sherry 2013 [39]), (Sherry \& Katz 2013 64]).
} 
as a theory of the formation of scientific concepts, pointing out the salient differences between 'Aristotelian' substantial concepts of common sense and the functional or relational concepts of modern science. In Section 3, we consider two neo-Kantian attempts to elucidate the progressive conceptual evolution in science with the help of mathematical metaphors, to wit, Natorp's equational metaphor and Cassirer's Cauchy metaphor. In Section 4, we discuss several attempts by the members of the Marburg school to elucidate some of Cohen's notoriously obscure theses on the "essence" of the infinitesimal. The issue of the closing Section 5 is Cassirer's road from infinitesimal to functional concepts in his mature philosophy of science and mathematics.

\section{Neo-Kantian Philosophy of Science and Mathematics}

To set the stage, let us review some historical and philosophical background. Following the collapse of German idealism after Hegel's death in 1831, German philosophy once again returned to Kant (cf. Coffa 1991 [12]). Such a reorientation did not, however, result in a new Kantian orthodoxy. Rather, the emerging neo-Kantian philosophy, subscribing to the maxim "With Kant Beyond Kant" (Otto Liebmann 1865 [45]) adopted some of Kant's ideas and at the same time came to criticize the master. The most important currents of neo-Kantianism were the so-called Marburg school founded by Hermann Cohen, and the Southwest or Baden school founded by Wilhelm Windelband and Heinrich Rickert. With some oversimplification one may say that the Southwest school was mainly interested in matters of Geisteswissenschaften, while the members of the Marburg school were mainly engaged in the task of a philosophical understanding of mathematics and the sciences. We will therefore concentrate on the neo-Kantian doctrines of the Marburg school and its contributions to a philosophical understanding of the problems posed by infinitesimals, limits, and related concepts.

Baldly characterizing an account in epistemology or philosophy of science as neo-Kantian may suggest that such an approach is rather similar to Kant's. This would be an error. All neo-Kantians agreed that Kant's philosophy was a promising starting point for modern epistemology and philosophy of science, but not a doctrine that had to be followed literally. Not surprisingly, they vigorously disagreed concerning the best way to go "beyond Kant". In this paper, however, we will not dwell on the issue of whether or not the Marburg neo-Kantian interpretation of Kant did true justice to Kant (cf. Friedman 2000 [23] and M. Kühn 2010 [40]), as our main topic is neo-Kantian rather than Kantian philosophy of science and mathematics. 
An authoritative survey of the essence of the Marburg neo-Kantianism was published by Natorp on the occasion of Cohen's seventieth birthday in 1912, in the prestigious journal Kant-Studien. The article Kant und die Marburger Schule [54] can be considered as a kind of official position paper of the Marburg school. More precisely, Natorp sought to present the Marburg current as the true heir, although not an epigone, of Kant's original philosophy. This endeavor had two parts. On the one hand, he emphasized the salient differences among the Marburg school's neo-Kantianism, Kantian orthodoxy, and rival contemporary philosophical currents such as the Baden school and neo-Hegelianism; on the other hand, he pointed out that the Marburg school preserved the true essence of Kant's doctrines.

2.1. The Transcendental Method. Natorp emphasized that for the Marburg school, the true core of Kant's philosophy was the transcendental method ([54, p. 194f]) 2 Everything in Kant's system that did not fit well with this method had to be given up by true Kantians. The transcendental method ${ }^{3}$ deals with the problem of the possibility of scientific experience. More precisely, pursuing the 'transcendental method' as the universal method of philosophy is contingent upon two requirements:

The first is a solid contact with the established facts of science, ethics, arts, and religion. Philosophy cannot breathe in empty space of pure thought, where reason aims to fly high only on the wings of speculative ideas. ... The place of philosophy ... is the fertile lowlands of experience in a broad sense, i.e., it seeks to take roots in the entire creative work of culture (science, politics, art, religion) ...

The second, decisive requirement of the transcendental method is to provide for these cultural facts (science, ethics, art, ...) their conditions of possibility. In other words, philosophy, by following the transcendental method, has to exhibit and to elaborate the lawful

\footnotetext{
${ }^{2}$ Whether or not the 'transcendental method' à la Natorp was also the core of Kant's philosophy need not concern us here. Indeed, many renowned Kant scholars deny this.

"The method was called "transcendental" since it went beyond the cognition that is immediately directed onto the objects. A more detailed discussion appears in the main text at footnote 8 .

${ }^{4}$ The original German "gesetzmässig" is a key term of neo-Kantian philosophy of science and difficult to translate. Its meanings range from order-generating to exhibiting regularity.
} 
ground ("Gesetzesgrund") or logos of those creative acts of culture (Natorp [54, p. 197]).

Restricting our attention to the cultural fact of science, we may restate Natorp's thesis in more modern terms by saying that philosophy of science in the transcendental mode has the task of rationally reconstructing the evolution of scientific knowledge and the conditions of its possibility.

The task of revealing the conditions of the possibility of scientific experience binds the Marburg 'transcendental method' to Kant's original 'transcendental logic', which, by definition, investigates how it is possible that our concepts are related to real objects. More precisely, transcendental logic in Kant's sense is concerned with the origin, the content, and the limits of experiential knowledge.

Pursuing the transcendental method, the "critical idealism" of the Marburg school is led to a genetic epistemology and theory of science that regards the ongoing process of scientific creativity as its essential feature, more so than its temporary results. Natorp put it as follows: Knowledge is always in the state of "becoming", it is never "closed" or "finished". Something non-conceptually "given", in particular something allegedly intuitively "given" cannot be accepted as such. A "given" is just another name for a problem to be solved.5

In other words, for the philosophers of the Marburg school the fact of science was a "fact of becoming" (Werdefaktum). Accordingly, the basic task of a truly "Kantian" philosophy of science was to make explicit the method of science as "the method of an infinite and unending creative evolution of reason" (Natorp [54, p. 200]).

The rejection of a non-conceptual given in any form brought the Marburg neo-Kantianism in open conflict with one of the cornerstones of Kant's epistemology, to wit, the dualism of conceptual understanding and intuition, as a "non-conceptually given". Indeed, as the Marburg neo-Kantians argued against Kant's original position, if one really follows the transcendental method in its true sense, then

it is virtually impossible, as Kant does, to maintain this dualism of epistemic factors if one takes seriously the core idea of the transcendental method. ([54], 201)

\footnotetext{
${ }^{5}$ As a then popular neo-Kantian pun put it: An object is not gegeben but aufgegeben. This pun loses its effectiveness in English: it claims that an object is not "given" (gegeben) but "posed" (aufgegeben) as a problem. The Russian equivalent dan/zadan appears in a recent collection of essays on neo-Kantianism, where it is mentioned in an essay by Sebastian Luft as translated by N. Dmitrieva [30, p. 121], and in an essay by T. B. Dlugach [30, p. 224].
} 
For the Marburg neo-Kantians, in contrast to Kant, both the Kantian categories and his forms of sensibility of space and time were purely conceptual. Kant's sharp separation of understanding and sensibility as two complementary faculties of the mind had to be given up.

Since Kant's original transcendental logic as a logic of the possibility of experience was closely related to the forms of sensibility, the Marburg neo-Kantians were led to give up the distinction between formal (deductive) logic and transcendental logic. For them, there was only one logic, namely, the logic of the transcendental method as the comprehensive logic of the conditions of the possibility of scientific experience (cf. Heis 2010 [33, p. 389]).

2.2. Concepts in Mathematics and Science. The emphasis on the evolving character of scientific knowledge gave the issue of the evolution of scientific concepts a central position in the Marburg philosophy of science. In particular, to Cassirer, this meant that philosophy of science had to investigate the common evolution of scientific and mathematical concepts firmly planted in the course of their historical development. From the Marburg perspective, these two conceptual developments were two aspects of the same problem. In Substanzbegriff und Funktionsbegriff 1910 [7] (henceforth, SF) Cassirer wrote:

[We should consider] physical concepts no longer for themselves but, as it were, in their natural genealogy, in connection with the mathematical concepts. In fact, the physical concepts only carry forward the process that is begun in the mathematical concepts, and which here [in mathematics-the authors] gains full clarity. The meaning of the mathematical concept cannot be comprehended, as long as we seek any sort of presentational correlate for it in the given; the meaning only appears when we recognize the concept as the expression of a pure relation, upon which rests the unity and continuous connection of the members of a manifold. The function of a physical concept also is first evident in this interpretation. (SF 1910 [7. pp. 219-220]; p. 166 in the 1953 edition)

Note that Cassirer employed terms such as "continuous", "connection", and "manifold" not only in their strict mathematical sense, but also in a metaphorical sense. Such usage of philosophical and scientific concepts was typical of Cassirer's thought throughout his entire philosophical career (see Orth 1996 [55]). Following the Husserl scholar Eugen Fink, Orth refers to concepts used in such a "metaphorical" way, as "operative concepts" as opposed to "thematic concepts". Operative 
concepts are concepts based on "intellectual schemata" without being fully explicit. They serve as "orientations in a conceptual field" and are of a metaphorical character. As Orth points out, Cassirer had at his disposal a particularly rich supply of operative concepts stemming from the (Kantian) philosophical tradition and from contemporary science, in particular physics and mathematics (cf. Orth, ibid. 111). Continuity and its relatives were among Cassirer's favored operative concepts.

In contrast to many currents of contemporary philosophy of science, the philosophers of the Marburg school regarded philosophy of the sciences and philosophy of mathematics as being of the same ilk, namely, as a study of relational concepts. It may be considered as a pleasing confirmation of Cassirer's unified approach to mathematical and physical concepts that one of the great mathematicians of the 20th century, Hermann Weyl, subscribed to a similar view, quite independently of Cassirer and apparently unaware of the similarity of convictions.

Weyl sought to overcome the deficiencies of a purely formal conception of mathematics such as Hilbert's without being forced to build mathematics on a restricted base of a Brouwerian intuitionism. Weyl therefore proposed to seek help from physics. More precisely, he considered theoretical physics as the guiding example of a kind of knowledge endowed with a meaning completely dfferent from that of the common sense or phenomenal meaning. Thus, in order to endow the symbols of mathematics with a meaning, Weyl saw only one possibility:

... [to] completely fuse mathematics with physics and assume that the mathematical concepts of number, function, etc. (or Hilbert's symbols) generally partake in the theoretical construction of reality in the same way as the concepts of energy, gravitation, electron, etc. (Weyl 1925 [66, p. 30])

Weyl clung to this thesis of the essential similarity of conceptualization in mathematics and the sciences till the end of his life. The conclusion he reached in his late essay A Half Century of Mathematics is fully in line with the neo-Kantian approach:

It is pretty clear that our theory of the physical world is not a description of the phenomena as we perceive them, but [rather] is a bold symbolic construction. However, one may be surprised to learn that even mathematics shares this character. (Weyl 1951 [67, p. 553])

This agreement between Weyl and the Marburg school is all the more remarkable since Weyl arrived at it from a rather different philosophical 
background: he was influenced mainly by Husserl's phenomenology, and never had shown in his mature age any affinity to Kantian or neoKantian philosophy.

2.3. Substantive versus Relational Concepts. After these general remarks on the Marburg account of mathematical and scientific concepts, it may be expedient to consider some concrete examples in detail. Thereby we can hope to illustrate what the Marburg philosophers, in particular Cassirer, intended to convey by their thesis of the relational (or functional) character of scientific concepts. Let us start with an elementary example. At first view, which is sometimes called the Aristotelian one, there appears to be a close analogy between common-sense concepts such as rock and mathematical concepts such as number or triangle, in that the concept rock corresponds to the class of all empirical entities that are rocks, i.e. the class of all entities that have all the properties a rock is assumed to have; and similarly, the mathematical concept number is said to correspond to the class of all mathematical "entities" that are numbers, i.e. the class of entities that have all the properties numbers are assumed to have.

Cassirer rejected such an analogy. According to him, the unity of mathematical and scientific concepts was not to be found in any fixed group of properties, but rather in the rules $\sqrt[6]{6}$ wich represented, in a lawful way, the mere diversity of objects that "fall under the concepts" as their cases (i.e., instantiations). Elementary examples of relational scientific concepts in this sense are mathematical formulas that describe arithmetic series (i.e., sequences) such as $1,3,6,10, \ldots$ For such a series, the "construction of unity" is provided by a formula that describes their generation according to some general law. For instance, the series $1,3,6,10, \ldots$ is characterized by the law that the difference of the differences of its consecutive members is always 1 . This fact is succinctly expressed by the formula $a(n)=n(n+1) / 2, n \in \mathbb{N}$. The members of such a series do not have a common property (in any ordinary sense of property) but appear as cases of a common functional law.

More generally, Cassirer considered the formulas of mathematics, physics, and chemistry as paradigmatic examples of relational scientific concepts since they brought singular facts into a lawful context. Algebraic equations of geometric curves provide somewhat less elementary examples. Such equations can be used to describe the movement of

\footnotetext{
${ }^{6}$ Mathematically speaking, the dichotomy is between properties, i.e., unary relations, and binary (and higher) relations.
} 
material bodies. More precisely, they are conceptual devices for embedding the individual perceived positions of a body in a continuous, even, smooth trajectory. Continuity, smoothness and other concepts of the infinitesimal calculus, are, however, highly theoretical 'ideal' concepts. The embedding of singular data into a continuous or smooth trajectory is anchored in a complex web of crucial idealizing assumptions.

For the Marburg neo-Kantians, the indispensable role of idealizations such as continuity and smoothness for modern science demonstrated that the real could be understood only through the ideal. To Cohen, this translated into a statement that the infinitesimal was a core concept of any truly modern logic of science. Cohen appears to have assumed that the notion of the infinitesimal necessarily underlies the concepts of continuity and smoothness (which is technically speaking not the case from the viewpoint of modern $\epsilon, \delta$ definitions of continuity and smoothness). In the opening chapter Infinitesimal-Analysis of his Logik der reinen Erkenntniss he explicitly contended:

If logic is to be a logic of science, i.e., a logic of the mathematized natural sciences, then it must be primarily the logic of the principle of the infinitesimal. If this is not the case and this core principle does not occupy centre stage, then logic itself still hasn't gained its proper centre, it still belongs to the past. The new scientific thought is that which since Galileo, Leibniz, and Newton has become systematically efficient [and for which the infinitesimal does play a fundamental role-the authors]. (Cohen 1902 [14, p. 31])

According to Cohen, the Leibnizian principle of continuity was the key that had opened the gate toward such a truly modern logic of the infinitesimal (see Section 41). Regrettably, however, later generations of philosophers and scientists had not faithfully followed Leibniz' lead. Therefore, a logic of the infinitesimal was still in its infancy. It was incumbent upon the Marburg school to develop the foundations of a working logic of the infinitesimal.

A paradigmatic example of a relational concept in physics was for Cassirer the concept of energy. The utility of the concept of energy is not to describe any new class of objects, alongside the already known physical objects such as light and heat, electricity and magnetism. Rather, it signifies only an objective lawful correlation, in which all these "objects" stand. The meaning of the concept of energy resides in the equations that it establishes among different kinds of events and processes. Energy in the sense of modern science is not an object in 
the traditional sense, but a unifying perspective that sheds light on a manifold of experiences.

This is rendered most evident by the functional identity of potential and kinetic energy through which states are identified with temporal processes:

The two [moments of kinetic and potential energy] are "the same" not because they share any objective property, but because they occur as members of the same causal equation, and thus can be substituted for each other from the standpoint of pure magnitude (SF 1910 [7. p. 264-265], (1953, p. 199))

Energy cannot be understood as the conceptual counterpart of something empirical out there. Rather, it is to be understood as an ordergenerating principle. In this respect it resembles the notion of number by which we make the sensuous manifold unitary and uniform in conception (cf. SF $(1910,252),(1953,189))$. In contrast to the concept of number, the concept of energy is a genuine concept of the empirical sciences. Hence, since "number" and "energy" both served as order-generating principles in essentially the same manner, this was considered as another argument in favor of the Marburg thesis that mathematics and mathematized empirical sciences followed the same rules of one and the same transcendental logic. The concept of energy shows that in modern science the allegedly objective "things" of common sense and traditional metaphysics are replaced by a web of mathematically formulated relations that yield objectivity to scientific knowledge. Thereby the notorious Kantian "things-in-themselves" can be dispensed with:

We need, not the objectivity of absolute things, but rather the objective determinateness of the method of experience. [SF, (1910, 428), (1953, 322)]

Characterizing scientific knowledge by idealizing functional relations reveals that it does not aim at a description of how the world "really is". The concepts of modern science are not the mental images of certain pre-existing objects; rather they are tools that offer new unifying perspectives as he elaborated in full detail in his magnum opus Philosophy of Symbolic Forms (Cassirer 1923-1929, 1953-1957 [9]) (henceforth PSF), in particular in the third volume that takes up many issues of $\mathrm{SF}$.

Ideal gases, ideal fluids, etc. are not limiting cases approximated by the more or less homogenous gases or the more or less ideal fluids found in nature. Rather, idealizing concepts such as perfect gases or 
perfect fluids have an epistemological role. They provide conceptual perspectives that allow the formulation of general relational laws and thereby they help to make sense of reality as a manifold of experiences.

Cassirer described this theoretical unification of the scattered data of sensations as an embedding of an incomplete empirical manifold of sensations in a completed conceptual manifold. Typically, such embeddings can be carried out in a variety of ways. In contrast to Kant, for the neo-Kantian Cassirer there were no fixed forms that determined how this process was to be carried out. Rather, the ever-growing variety of conceptual completions of our experiences is revealed in the historical evolution of science itself. For Cassirer, the paradigmatic example of such a conceptual completion was Dedekind's completion of the rational numbers $\mathbb{Q}$ to the real numbers $\mathbb{R}$. The essential point of this completion was not that some "ideal" numbers were "added" to the already existing rational numbers, but that the relational system $\mathbb{R}$ of real numbers provided us with a new conceptual perspective to "see" more clearly the conceptual essence of the rational numbers $\mathbb{Q}$ themselves (cf. PSF III, 392).

Although the processes of concept formation in mathematics and physics are similar, they are not identical. After all, there is a difference between mathematics and physics, and philosophy of science has the task of elucidating this difference. Roughly, Cassirer considered conceptualization in mathematics as a simplified version of conceptualization in physics:

In contrast to the mathematical concept, however, in empirical science the characteristic difference emerges that the construction which within mathematics arrives at a fixed end 7 remains in principle incompletable within experience. But no matter how many "strata" of relations we may superimpose on each other, and however close we may come to all particular circumstances of the real process, nevertheless there is always the possibility that some relevant factor in the total result has not

\footnotetext{
${ }^{7}$ Here Cassirer appears to express the view that in mathematics the construction arrives at a fixed end. This reading seems hardly compatible with his general neoKantian outlook according to which the essence of science resides in its unending evolution. A more plausible reading of Cassirer here would be that he contended that mathematical concepts are relatively fixed with respect to empirical concepts, just as the elements of an infinite convergent series of numbers are fixed although the series itself may not reach a fixed limit point in finitely many steps.
} 
been calculated and will only be discovered with the further progress of experimental analysis (Cassirer 1910 [7, p. 337], $(1953,254))$.

Factual and theoretical components of scientific knowledge cannot be neatly separated. In a scientific theory "real" and "non-real" components are inextricably interwoven. Not a single concept is confronted with reality but a whole system of concepts.

\section{Two Guiding Metaphors of neo-Kantian philosophy of SCIENCE}

In line with the essence of the transcendental method that conceived of science "as an infinite and unending creative evolution of reason", the philosophers of the Marburg school considered empirical or mathematical concepts (or theories as systems of concepts) as stages in an ongoing process of an unending conceptual approximation. Accordingly, the task of philosophy of science was to investigate the conditions of possibility for such an evolution. Remarkably, for this endeavor the Marburg neo-Kantians heavily relied on that science whose conceptual evolution they sought to elucidate, to wit, mathematics. In other words, both Natorp and Cassirer, each in his own way, sought to tap the resources of mathematics to elucidate the structure of the conceptual evolution of the sciences. To this end, they introduced certain mathematical metaphors, by exploiting the mathematical concepts of approximation and convergence for which the concept of the infinite played an essential role.

3.1. Natorp's Knowledge Equation. Perhaps the best-known of these metaphors in the hightime of neo-Kantian philosophy was Natorp's "equational metaphor" that compared the evolution of science with the solution of a numerical equation. According to it, coming to know an object ("Erkenntnisobjekt") was analogous to the process of solving a numerical equation. To be specific, the reader may keep in mind a specific equation such as $x^{3}-x^{2}+x-1=0$.

In line with Natorp's didactic intentions this equation has been chosen to convey several ideas concerning knowledge and its objects. First, the fact that it has several different solutions indicates that the process of research may not lead to unique results. Furthermore, the fact that two of its solutions are imaginary reflects the fact that the research process may lead to an expansion of the original fundamental concepts one started with. Note that the admission of complex numbers as solutions transcends the conceptual space in which the equation was formulated, since its coefficients are all integers. What is still missing 
in this metaphor is the "infinite character" of the knowledge equation. Natorp was aware of this shortcoming and tried to remedy it (see below).

According to Natorp, the object of knowledge may be considered as an "unknown $x$ of the knowledge equation":

If the object is to be the $x$ of the equation of inquiry, then it must be possible to determine the meaning of this $x$ by the nature of this equation (i.e., the inquiry itself) in relation to its known factors (our fundamental concepts). From this it must follow whether and in what sense the solution of this problem is possible for us. This is the very idea of the transcendental or critical method (Natorp 1903 [52, p. 10]).

Natorp added the following further elucidations. The transcendental method did not aim to extend our knowledge beyond the limits of the scientific method. Rather, it sought to clarify the limits of scientific knowledge. It was called "transcendental" since it went beyond the cognition that is immediately directed onto the objects, but aimed to obtain information about the general direction of the path to be taken 8 It did not provide us with any specific knowledge about an object beyond experience. Hence, following the established Kantian terminology it was transcendental, but not transcendent.

Both Natorp and all his fellow philosophers of the Marburg school viewed the object of knowledge, not as an unproblematic starting point of the ongoing process of scientific investigation, but rather as its limit. This object was a problem to be solved. In its various versions, this equational account of knowledge can be found in virtually all of $\mathrm{Na}$ torp's epistemological writings. One might object that Natorp's equational model of scientific cognition is far too simple in the sense that the empirical objects hardly ever show up as solutions of a finite equation such as the one considered above. It seems hardly plausible that physical entities such as "proton" or "quark" fit in the conceptual framework of one physical theory without remainder.

Natorp did not ignore this difficulty and complemented his equational account so as to counter this objection. Elaborating the equational model, he pointed out that the object of knowledge was not simply a problem ("aufgegeben") but an infinite problem that could

\footnotetext{
${ }^{8}$ Here we elaborate further on the term "transcendental" as discussed in footnote 3 .
} 
be solved in finite time only approximately by finite creatures like ourselves. He thus sought to elude the trap of an overstated Hegelian rationalism:

Although we conceive of the object of knowledge $(=x)$, similarly ${ }^{9}$ as Hegel does, only in relation to the functions of knowledge itself, and consider it ... as the $x$ of the equation of knowledge, ... we have understood that this "equation" is of such a kind that it leads to an infinite calculation. This means that the $x$ is never fully determined by the parameters $a, b, c \ldots$ of the equation. Moreover, the sequence of the parameters ... is to be thought of as being not "closed" but rather extendable further and further. (Natorp 1912 [54, pp. 211-212])

3.2. Cassirer's Convergent Series. As a second example of the usage of mathematical metaphors for the elucidation of the meaning of philosophical ideas, let us now have a closer look at how Cassirer conceptualized the guiding idea of the Marburg school, to the effect that the evolution of scientific knowledge could be understood as a conceptual approximation process. In contrast to Natorp, Cassirer insisted that this approximation was not assumed to converge to an externally given limit, but rather as defined by a general internal rule, as is done in the arithmetic of rational numbers. To give a mathematical model for such a conception of the evolution of science, we can characterize a rational sequence such as $3.1,3.14,3.141,3.1415, \ldots$ as being convergent, obviously without relying on an assumed existence of an element in $\mathbb{Q}$ to which it would converge.

Cassirer used this elementary mathematical insight to illustrate his thesis that one may meaningfully speak of the convergence of scientific theories without assuming that there is a fixed reality "out there" to which the sequence of our theories is expected to converge. To be specific, consider a sequence of positive numbers such as $(1 / n)$ that converges to 0 . This may apparently suggest that a convergent series 10 of concepts (or theories) converges to some ultimate external entity (or "reality"), just as the arithmetical series $(1 / n)$ converges to the real number 0 external to it, with 0 itself not being a member of the

\footnotetext{
${ }^{9} \mathrm{~A}$ common criticism of the Marburg school's epistemology was that it was dangerously close to an overstated Hegelian rationalism. Hence Natorp, although he had to admit some similarity with Hegel, was at pains to distance the Marburg neo-Kantianism from any sort of Hegelian rationalism.

${ }^{10}$ Cassirer used the term equivalent to "series" for the mathematical entity usually referred to as a "sequence". We have retained Cassirer's terminology.
} 
series $(1 / n)$. Cassirer vigorously rejected such a realist "exterior" interpretation:

The system (Zusammenhang) and the convergence of the series take the place of an external standard of reality. Both system and convergence can be established and determined, analogously to arithmetic, entirely by comparison of the serial members and by the general rule, which they follow in their progress. (Cassirer 1910 [7, p. 426], $(1953,321))$

As already 19th century mathematics had taught us, in order to be able to speak meaningfully about a convergent sequence of numbers, it is not necessary to assume that there "really" is a number to which the sequence converges. Rather, an arithmetical series can be defined as convergent if it satisfies an internal requirement that can be formulated without reference to a possibly inexistent external limit. Such an internal requirement is provided by Cauchy's criterion.

Mathematicians have pushed this "internalization" of the concept of convergence even further. As is well known, the "gappiness" of the rational numbers $\mathbb{Q}$ (residing in the absence of limit points of certain Cauchy-convergent sequences) may be overcome by completing $\mathbb{Q}$ in an appropiate way. More precisely, one can embed the rationals $\mathbb{Q}$ into a set $C(\mathbb{Q})$ of appropriately defined equivalence classes of Cauchy sequences. Thereby it can be ensured that in the new completed realm $C(\mathbb{Q})$ that englobes the rational numbers $\mathbb{Q}$ as a part, every Cauchy sequence has a limit point. From this viewpoint, a real number is an encapsulation, or reification, of the concept of convergence of a Cauchy sequence.

Cassirer took these mathematical constructions to be more than mere technicalities. He considered them as the pattern for his internally defined account of the continuous evolution of scientific knowledge:

No single astronomical system, the Copernican any more than the Ptolemaic, can be taken as the expression of the true cosmic order, but only the totality of these systems as they unfold continuously according to a definite connection. (Cassirer 1910 [7, p. 427], (SF, 322))

In other words, for Cassirer the "true cosmic order" was not given by a single theory but by a convergent series of theories. He did not assert that our theories ontologically converge to a mind-independent realm of substantial things as the substrate of a "final" theory. His notion of theoretical convergence was epistemological rather than ontological. He understood the approximation of theories essentially as 
an epistemological progress within which the historical progression of our theories continually approximates, but never reaches, any ideally complete mathematical representation of the phenomena. Such an ideal representation is not waiting "out there" to be approximated; rather, it resides in the reification of the approximation process that comes into being through this very process itself. In other words, in the course of its history science completes itself, so to speak, analogously as a convergent sequence without limit gives rise to a corresponding sequence with limit point in a suitably completed domain. For Cassirer, the paradigmatic example of such a completion was Dedekind's completion of the rationals to the real numbers. But for him, the significance of Dedekind's construction went beyond developing a more abstract version of unending decimal expansions. According to him, idealizing completions were the essence of the modern empirical and mathematical sciences (cf. Mormann 2008 [50]).

3.3. Idealisations, completions, and infinitesimals. The "completionfriendly" perspective of the Marburg school on the conceptual evolution of science had important consequences for matters infinitesimal. With respect to completions of number systems by infinitesimals, the thesis of the "incompletability" of the conceptual evolution in science and mathematics suggested that Cassirer's account (and that of Marburg neo-Kantianism in general) had no built-in source of resistance to further ontological extensions beyond the rational and the real numbers. On the contrary, according to its own rules, the neo-Kantian approach would have welcomed the advent of the hyperreals à la Edwin Hewitt [35] and Abraham Robinson [58, 59] and related developments. As we shall see in the following sections, the insistence on the openness of the evolution of mathematical and physical concepts brought the Marburg philosophers in conflict with mathematicians and logicians such as Cantor and Russell, who adhered to more realist accounts that considered concepts as more or less direct descriptions of what there is (in the empirical or in an ideal realm), instead of conceiving of them as epistemological tools for progressing in the task of making sense of some aspects of the world. According to Cassirer, both empiricism 
à la Bacon and Berkeley, and naive realism 11 with respect to "ideal" mathematical entities, à la Cantor and Russell, must be rejected:

For the existence of the ideal, which can alone be critically affirmed and advocated, means nothing more than the objective logical necessity of idealization. (SF 1910 [7, p. 170], (1953, 129))

And again:

The relation beween the theoretical and factual elements at the basis of physics cannot be described in this simple way. It is a much more complex relation, it is a peculiar interweaving and mutual interpenetration of these two elements, that prevails in the actual structure of science and calls for clearer expression logically of the relation between principle and fact. (Cassirer (1910, 172), (1953, 130))

According to Cassirer, no scientific theory directly relates to the facts of perception. Rather, such a theory relates to the ideal limits, which we substitute for the facts of perception. Thus, we investigate the impact of bodies by regarding the masses, which affect each other, as perfectly elastic or inelastic. We study perfect fluids even though no such are to be found. In other words, Cassirer sought to present his "Critical Idealism" as a theoretical framework that overcame both a naive empiricism and a misled platonist idealism.

For the Marburg neo-Kantians, who had always emphasized the essential unity of mathematics and empirical science, the new relational logic (Frege, Peano, Russell and others; see e.g., Gillies [28]) was part and parcel of a single comprehensive transcendental logic of science that was emerging in the course of the history of science. For them, it was a fundamental philosophical mistake of the 'logicism' of Frege and Russell to ignore its transcendental character in conceiving of it as a purely formal device neatly separated from the empirical realm.

\footnotetext{
11 "Realism" is taken here as an unsaturated term, i.e., questions of realism arise with respect to a certain subject matter, e.g., realism with respect to atoms, values, mathematical objects, possible worlds, causality, or macroscopic material objects. Cantor and Russell were realists with respect to mathematical entities. Other names for this sort of realism are "platonism", "platonist realism", or even "platonist idealism". Cassirer, a self-proclaimed "critical idealist" was not a partisan of platonist idealism. On the contrary, he vigorously criticized it.
} 
For the neo-Kantians, this was just another example of a philosophically untenable dualism, analogous to the Kantian dualism between the conceptual and the sensual 12

One of Cassirer's criticisms of Russell's philosophy of logic was that it succumbed to a naive "platonizing idealism" (see also J. Heis [33, p. 386]) since it insisted on a strict separation between the logicomathematical conceptual realm, on the one hand, and the empirical realm, on the other. According to Cassirer, this stance expressed itself in an outdated dualistic metaphysics that was bound to lead into unsolvable, self-inflicted pseudo-problems (cf. SF 1910 [7, pp. 313, 359], (1959, pp. 237, 271)). For instance, the applicability of mathematics in the mathematized natural sciences became an unfathomable mystery 13

\section{Three ATtempts to MAKe SEnSE OF COHEN}

The philosophers of Marburg neo-Kantianism considered themselves consciously as members of a well-defined school under the leadership of Hermann Cohen as the school's founder. Even when they held differing opinions concerning a philosophical question, they sought to minimize their differences vis-à-vis outsiders. An important example of such "school discipline" concerns the concept of infinitesimals and how it was dealt with in the treatises of the school's leader Cohen.

Even for sympathetic readers, it is often difficult to make sense of Cohen's writings. Such a difficulty is not limited to contemporary readers accustomed to doing philosophy in a more analytic style. Already the young Husserl in 1886 complained in a letter to Brentano that Cohen's allegedly "scientific philosophy" was actually nothing but "nonsensical profundity" or "profound nonsense" (see Mohanty 2008 [47, p. 3]). As evidence for this strong claim Husserl took Cohen's theory of the principle of continuity; see Das Prinzip der Infinitesimalmethode und seine Geschichte (The Principle of the infinitesimal method and its history) (henceforth Prinzip) 1883 [13, §40ff].

We argue that Husserl's radical verdict was not entirely justified. For this purpose we will rely on three different attempts to make sense of Cohen's "scientific philosophy" undertaken by three members of the Marburg school, namely Ernst Cassirer, Dimitry Gawronsky, and Paul Natorp. The works involved are Cassirer's Leibniz' System (1902), Substanzbegriff and Funktionsbegriff, SF (1910), Gawronsky's Das Urteil

\footnotetext{
${ }^{12}$ See Section 2

${ }^{13}$ Perhaps Wigner put forward the most influential plea for this attitude in terms of "the unreasonable effectiveness of mathematics in the natural sciences" (cf. 68 and 29$]$ ).
} 
der Realität (1910), and Natorp's Die logischen Grundlagen der exakten Wissenschaft (1910). As we will see, it reveals that the evolving thought of the Marburg school about infinitesimals and related concepts was not monolithic and without cracks. This is not surprising, since Cohen's Prinzip and Cassirer's SF are separated in time by almost thirty years, not to mention the rather different technical styles and the scientific background of their authors. Thus, Cohen's education in logic did not correspond to the state of the art at the beginning of the 20th century. He apparently never took proper notice of Frege, Russell, or any other contemporary logician. On the other hand, Cassirer, Gawronsky, and Natorp were aware at least partially of the new developments in logic and mathematics, and sought to adapt the Marburg school's philosophical stance to the new circumstances (cf. Cassirer 1907 [6]).

4.1. The Point of departure: Cohen's Prinzip. Cohen treated the issue of the infinitesimal first in Das Prinzip der Infinitesimalmethode und seine Geschichte and later, in Logik der reinen Erkenntnis. The Logik may be considered as a continuation and philosophical elaboration of Prinzip. The most significant difference is that the last traces of any sort of Kantian 'intuition' in the constitution of infinitesimals are eliminated. Pure thought 14 and pure thought alone, takes care of matters infinitesimal. Cohen even went so far as to contend that the infinitesimal was to be considered as the most important and most typical issue of pure thought, überhaupt 15 In his Logik, Cohen appears to assume that the reader has read and digested the argumentation of Prinzip. That is to say, mathematically there is nothing new in Logik that cannot be found already (usually more fully elaborated) in Prinzip. The main purpose of Logik is rather to explicate Cohen's account of the philosophical or metaphysical presuppositions and ramifications of pure thought, centering on the notion of the infinitesimal.

In Prinzip, Cohen still sought to establish the existence of infinitesimals with the aid of an intuition in the sense of Kant, while in Logik any vestige of Kantian intuition had vanished. According to mature Marburg neo-Kantianism, intuitions played no role in scientific knowledge. This must not be misunderstood: Cohen's target was Kantian

\footnotetext{
${ }^{14}$ The expression pure thought ("reines Denken") was the technical term designed by the neo-Kantians as the successor concept of the two separate components of Kantian epistemology, to wit, "concepts" and "intuitions".

${ }^{15}$ According to Cohen transcendental logic was the logic of the infinitesimal (see Subsection 2.3). Infinitesimals provided the key example of how the real was clarified by the ideal.
} 
intuition, not intuition in the everyday understanding of the term. Recall that Kant distinguished between two basic components of human representations, to wit, concepts and intuitions. These correspond to two essentially different cognitive faculties: understanding and sensibility. Only if these faculties are united can knowledge be achieved. The neo-Kantians argued against this two-tiered Kantian epistemology, not against the common-sense claim that for the individual scientist, "intuitions" may play an important role in the context of discovery.

Though this may not be crystal-clear already from the first pages of Prinzip, in later works, in particular in Logik, Cohen repeatedly emphasized this difference between orthodox Kantianism and Marburg neo-Kantianism. The rejection of the significance of "Kantian intuition" for scientific knowledge was, as is well-known, a common trait of all members of the school. Hence, critizising Cohen for relating the concept of the infinitesimal to some sort of Kantian intuition would be a gross misunderstanding. Another more subtle, albeit quite common misconception would be to ascribe to Cohen the claim that he sought to locate the "problem of the infinitesimal" in the realm of a psychologistically conceived epistemology (Erkenntnistheorie).

Already in Prinzip, Cohen had insisted that the problem of the infinitesimal could be properly treated only in what he referred to as the logic of science a.k.a. transcendental logic. According to him "the logic of science must be the logic of the principle of infinitesimal calculus" (cf. Logik, already quoted in Section 2.3). He was eager to point out that a glance at the literature revealed that the logic of his day had not yet recognized the decisive logical significance of the infinitesimal principle. In other words, and to give it a more personal twist, in Logik he admitted that his Prinzip had not yet found the recognition it deserved.

This situation did not change in the ensuing decades. Thus, in the otherwise rather comprehensive survey by Haaparanta, The Relation between Logic and Philosophy, 1874 - 1931 (Haaparanta 2009 [31]), Cohen's and, more generally, the Marburg account of logic as transcendental logic of science was completely ignored.

Cohen blamed Kant, to some extent, for this myopic conception of logic. According to him, Kant had failed properly to understand the role of the infinitesimal for a true critique of pure reason. Instead, he introduced pure sensitivity as a second ingredient of knowledge, whereby the independence of pure thought had been compromised (cf. Cohen (1902) p. 32)).

Let us now take a closer look at Cohen's general conception of logic. Cohen's conception incorporates both modern and obsolete ideas in a 
peculiar mixture. The first thing to note is that for Cohen logic is independent of anything else: logic is neither a branch of psychology nor a branch of linguistics. The laws of logic, i.e., the laws of "pure thought" are neither psychological laws nor grammatical laws. Rather, according to Cohen, the laws of logic constitute the core of pure thought. Thoughts in Cohen's sense should not be confused with "sensations" (Empfindungen) or individual mental representations (Vorstellungen). Cohen vigorously rejected any dependence of logic on another realm. For him, logic described the activity of pure thought that took place in the ongoing conceptual evolution of science: "The thought of logic is the thought of science. Thought constitutes the foundation of being" (Cohen 1902 [14, pp. 17, 18]).

Cohen's conception of logic is a far cry from any modern post-Fregean or post-Russellian conception of logic. There are no axioms, inferential rules or anything of that sort. Rather, his logic follows, at least superficially, quite closely the patterns of a Kantian (or even pre-Kantian) Urteilslogik (judgment logic). Thereby Cohen's Logik reveals a somewhat paradoxical relation to Kant. On the one hand, it can be read as a definitive parting of ways with Kantian orthodoxy, in particular by giving up the basic structure of the Kantian philosophical system, namely the distinction between the two pillars of pure logic and pure sensitivity. On the other hand, Cohen formally followed the architectonics of the transcendental logic of the Critique of Pure Reason when he mimicked in his Logik Kant's system of categories and judgments. In close analogy to Kant's pair of $4 \times 3$ schemata of categories and judgments, Cohen set up the following $4 \times 3$ schema distinguishing between four classes of judgments each consisting of three types of judgments:

- The judgments of laws of thought (Origin, Identity, Contradiction)

- The judgments of mathematics (Reality, Majority, Totality)

- The judgments of mathematized natural sciences (Substance, Law, Concept)

- The judgments of methodology (Possibility, Contingency, Necessity)

For the purposes of the present text, it is unnecessary to dwell upon Cohen's schema in full detail. But the following remarks may be in order. Superficially, Cohen's and Kant's tables of judgments are quite similar. Both exhibit the $4 \times 3$ schema, and, moreover, Cohen's "judgments of methodology" and virtually identical with Kant's "judgments of modality". Behind this formal similarity, however, are lurking deep 
conceptual differences. The first is that Kant's schema is based on what he called general logic, based on the standard formal logic of his time. In contrast, Cohen's table is deeply soaked with contentious assumptions of his account of transcendental logic. Moreover, it is not concerned with knowledge in general, but with knowledge of the mathematized natural sciences. In this respect, Cohen's epistemological perspective was considerably narrower than Kant's. Perhaps even more important is the difference between Kant and Cohen concerning the problem of how to conceive of the relation between the table of judgments and the corresponding table of categories. In Cohen's Logik, there is nothing that even remotely resembles Kant's famous transcendental deduction of the categories.

Kant invested immense efforts in the task of this deduction that resulted in a strict and rigid 1-1 correspondence between the $4 \times 3$ items of the table of judgments and the $4 \times 3$ items of the resulting a priori categories. In contrast, Cohen was content with the vague assertion that there was a mutual "correlation" between judgments and categories relying on the bland metaphorical explication that "the category is the aim of the judgment, and the judgment is the road to the category" (Logik, p. 47). In particular he gave up the 1-1 correspondence between categories and judgments, and allowed that every category might be contained in several judgments and every judgment might be contained in several categories. Actually, this vagueness and indecision is no co-incidence. Kant's categories were intended to be valid a priori, once and for all. There was no change or evolution in the categorical schema that Kant had set up in the Kritik. In contrast, Cohen repeatedly emphasized the evolving character ("Werdecharakter") of scientific knowledge, for instance, when he contended that the "truly creative elements of scientific thought reveal themselves in the history of scientific thought" (Logik, 46), but, obviously, this dynamic character of scientific knowledge was hardly compatible with a Kantian schema of fixed a priori categories.

For contemporary philosophy of science, Cohen's half-baked proposal of how to reconcile the categorical structure of scientific knowledge and its historical character can be of historical interest at best.

Nevertheless one may note that Cohen's problem, as we may call it, has remained on the agenda of virtually all accounts of philosophy of science that have been inspired by Kant up to this very day, as exemplified, for instance, by Reichenbach's reformulation of the Kantian $a$ priori in the 1920s, up to Michael Friedman's neo-neo-Kantian Dynamics of Reason (Friedman 1999 [22]) where the author seeks to reconcile 
historical and the a priori aspects of scientific knowledge as a synthesis of ideas taken from Kuhn, Cassirer, and Carnap.

As was already mentioned at the beginning of this section, we do not aim at an exhaustive treatment of Cohen's logic of judgments. Instead, we aim to shed some light on Cohen's often obscure analyses by consulting the writings of other members of the Marburg school, to wit, Ernst Cassirer, Dimitry Gawronsky, and Paul Natorp. These authors may be helpful in elucidating their master's thoughts, as all of them intended not to deviate from Cohen's ideas unless absolutely necessary. This does not mean, of course, that they actually provided faithful and accurate interpretations of Cohen's account. Nonetheless, the works of these three philosophers can be read as sympathetic readings that try to make the best out of Cohen.

4.2. Cassirer's Leibniz' System. Cassirer's first philosophical works were his 1899 dissertation Descartes' Kritik der Mathematischen und Naturwissenschaftlichen Erkenntnis 16 and his Leibniz' System in seinen wissenschaftlichen Grundlagen (Cassirer 1902 [5]).

Cassirer eventually published his Descartes and Leibniz texts together as one book proposing that Descartes may be conceived of as a forerunner of Leibniz. More precisely, according to Cassirer, Descartes and Leibniz may be considered as two stations of the long and winding road toward an idealistic conception of science. Provisionally, this was achieved in Kant's account; after Kant it found its contemporary expression in the philosophy of science, or the scientific philosophy, of the Marburg school. Cassirer's Leibniz' System is a 400 page long text. In its ten chapters Cassirer seeks to treat Leibniz' philosophical and scientific achievements within logic, mathematics, mechanics and metaphysics. The latter is understood in a broad sense, including Leibniz' reflections on issues such as "the problem of consciousness", "the problem of the individual", and "the concept of the individual in the system of Geisteswissenschaften." By far the largest (and for our purposes most interesting) chapter is the fourth, dealing with The Problem of Continuity. It comprises not less than 70 pages. In this section we will mainly concentrate on this chapter of Leibniz' System.

Leibniz' System is engaged in the ambitious task of presenting the conceptual evolution of modern science by presenting the achievements of the two geniuses of Descartes and Leibniz. The starting point was Descartes' overcoming of the medieval conception of science. According to Cassirer's fundamental thesis, the philosophical systems of these men could not be understood by separating them from their scientific

\footnotetext{
${ }^{16}$ Descartes' Kritik was published as the first part of Leibniz' System.
} 
achievements, to wit, analytical geometry in the case of Descartes, and the infinitesimal calculus in the case of Leibniz:

Through the discovery of analytic geometry Descartes lays the foundations for the modern way of scientific thinking, which finds its mature expression in the infinitesimal calculus. ... The synthesis of philosophy and science which is carried out thereby must not be conceived of as a mere juxtaposition. ... One must attempt to identify a common basis of these thoughts (Cassirer 1902 [5, pp. 1-2]).

Identifying such a common base will lead to a more profound understanding of the role of Descartes' system in the historical evolution of critical epistemological idealism and its continuation and completion in Leibniz and Kant and, one may add, in the idealism of the Marburg school. So much for Descartes. In the remainder of this section, we will concentrate on Cassirer's study of Leibniz as one of the most important early sources for the philosophy of science of the Marburg school.

According to Cassirer, for Leibniz mathematics was primarily an instrument of scientific research and a presupposition for the discovery of a new concept of nature, rather than an aim in itself (Cassirer 1902, 99). This became fully evident through the "new mathematics", i.e., the infinitesimal calculus. Following Cohen, Cassirer contended that for Leibniz' thought the concept of the infinitesimal was to be considered of fundamental importance, not only with respect to mathematics, but much more generally, also for Leibniz' philosophical understanding of the mathematized empirical sciences, a new concept of nature in general, and his metaphysics in general. Indeed, Leibniz' System is to be considered only as Cassirer's first attempt to contribute to this overall programme of a genuine Marburg philosophy of science inaugurated by Cohen's Das Prinzip der Infinitesimalmethode. While in Leibniz' System, Cassirer concentrated on the historical figures of Leibniz and Descartes, a few years later, he widened his perspective. In his monumental Das Erkenntnisproblem in der Neuzeit (The Problem of Knowledge) (Cassirer (1906-1950)) he became engaged in the huge project of writing a comprehensive intellectual history of ideas (Ideengeschichte) of Western thought of the modern period that he pursued during his entire lifetime in various forms: The last volume of Das Erkenntnisproblem, which eventually comprised four bulky tomes, was published only postumously in 1950 . 
Although in Leibniz' System young Cassirer still followed Cohen's lead in emphasizing the crucial importance of the infinitesimal for modern science and mathematics, the reader may notice an inclination toward relativizing its central role. This tendency gained momentum in later works such as Das Erkenntnisproblem (Cassirer 1906 - 1950), Substance and Function (Cassirer 1910 [7]), and The Philosophy of Symbolic Forms (Cassirer 1923 - 29). But already in Leibniz' System we find the sweeping thesis that the central concept of modern science is a concept of function 17 although it is not made clear how this assertion fits with the alleged primacy of the concept of the infinitesimal. These tensions became more evident in Substance and Function. Cassirer's failure to toe the party-line on the primacy of the concept of the infinitesimal over the concept of function was explicitly noted by Cohen; see Section 5 .

For Cassirer the really modern character of Leibniz' thought was encapsulated in the thesis that "the real is conditioned by the ideal." According to the Marburg idealism, this thesis was the key that opened the possibility of a truly modern philosophy of science, mathematics, and logic:

From this perspective we can really understand Leibniz' tendency to equate logic and mathematics in its true significance. This equation does not aim to constrict the rich content of mathematics in the form of traditional logic. Rather, it intends to bring about fundamental reformation for logic. Instead of being a theory of "thought forms", logic is to become a science of objective knowledge (gegenständliche Erkenntnis). This transformation is essentially due to its relation to mathematics: Mathematics turns out to be the necessary mediation between the ideal logical principles and the reality of nature. (Cassirer 1902 [5, p. 113])

For Cassirer and Cohen, Leibniz was the one who opened the gate for an idealist "transcendental logic" that later in the hands of Kant and the Marburg neo-Kantians was to become a "transcendental logic of objective knowledge".

4.3. The Continuity principle. The essential means for overcoming the traditional Aristotelian conception of logic as a theory of abstract "thought forms" toward a contentful theory of objective knowledge was

\footnotetext{
${ }^{17}$ Here Cassirer is not referring to the notion of function in a narrow mathematical sense, but rather to the functional (or relational) account of science according to which entities are secondary and functions or relations primary (see Subsection 2.2).
} 
said to be Leibniz' famous continuity principle. The continuity principle was considered as the most important device to unfold the general thesis that "the real is conditioned by the ideal", mentioned in Subsection 4.2. Consequently, the bulk of Leibniz' System was dedicated to the task of explicating this principle.

It should be kept in mind that for Cassirer and the Marburg philosophers in general, continuity was not a property that some things (or processes) have and others do not. Cassirer described its significance in the following terms: 18

For the philosophy before Leibniz, continuity was essentially nothing but the property of a thing or an attribute of a ready-made concept. When it was understood in this way, one could attempt to refute or to prove the claim that a certain thing or concept possessed it or lacked it. This holds true of the synechés of the Eleates till Descartes' concept of continuous space. Leibniz overcomes this stance. For him, the problem of continuity dissolves in the problem of "continuation". Continuity is no longer a characteristic of a thing, but rather that of a development; not of a concept, but of a method. (ibid., 153).

Therefore, it would perhaps have been more appropriate to refer to this principle as the principle of continuation rather than the principle of continuity. In order to be understood as a general conceptual achievement, continuation needs to be elaborated in the framework of a scientific methodology (wissenschaftliches Verfahren). At this point the infinitesimal and related concepts enter the stage. As Cassirer was eager to point out, the method of continuation first obtained its deeper "scientific" meaning in the domain of geometry,

where it designates the transition from point to line, from the line to the area and so on 19 Similarly, in mechanics and dynamics the method of continuation describes the

\footnotetext{
${ }^{18}$ Note that here Cassirer is using the concept of "continuity" as an "operative concept", as explained in Subsection 2.2

${ }^{19}$ From a purely mathematical viewpoint, Cassirer's formulation is a bit unfortunate here because it sounds as if one is still dealing with indivisibles rather than infinitesimals. The difference between them is that indivisibles were thought of as codimenion- 1 entities whereas infinitesimals were of the same dimension as the figure composed of them. This was the content of the major advance as accomplished by Roberval, Torricelli, Wallis, Leibniz, and others, as compared to earlier work by Archimedes and Cavalieri.
} 
relation of the material point to the structures of higher dimension.

In its true scientific generality the relation between an element and the structure that results from its continuation corresponds to the relation of a differential and its integral. In other words, the "continuation" is the methodical expression of the integration as a continuous summation of infinitesimal moments. (ibid., 153 - 154)

To a modern reader, Cassirer's "logical" thesis may sound utterly "metaphysical". Cassirer himself recognized that this general characterization of the relation between the infinitesimal and the real was in need of further clarification and scrutiny. For this task he proposed to have a closer look at Leibniz' foundations of the infinitesimal calculus. According to Cassirer, the key to understanding the true novelty of Leibniz' account resided in the Leibnizian concept of motion. The crucial point was not to view motion as an empirical concept stemming from the realm of empirical experience. For Leibniz, motion was always continuous motion, i.e., the expression of a unifying principle of conceptual construction. Leibnizian motion was not something empirically given, but something conceptually constructed (cf. ibid., 156). Invoking the idealist principle that "the real is clarified by the ideal", still another way of expressing this may be that, the concept of continuity, in Leibniz's view, belongs to the realm of the ideal.

Probably the most famous expression of this view can be found in Leibniz's letter to Varignon from which Cassirer quoted in his Leibniz' System (ibid., p. 188/189) and elsewhere:

... one can say in general that, though continuity is something ideal and there is never anything in nature with perfectly uniform parts, the real in turn, never ceases to be governed perfectly by the ideal and the abstract ... (Leibniz 1702 [42])

Later, in the same letter, Leibniz explicitly stated that not only continuity but also infinitesimals have the capacity to "govern the real perfectly":

So it can be said that infinites and infinitesimals are grounded in such a way that everything in geometry, even in nature, takes place af if they were perfect realities. (ibid.)

On the other hand, in the very same letter he asserted: 
I'm not myself persuaded that it is necessary to consider our infinities and infinitesimals as something other than ideal things (choses ideales) or wellfounded fictions (fictions bien fondées). (ibid.)

It is far from clear, however, how precisely the relation between "ideal things" and "(wellfounded) fictions" is to be thought and how infinitesimals as "fictions" could have this power of governing perfectly. This difficulty was already observed by Cassirer, who even contended that Leibniz's notion of a "fiction bien fondée" had an air of paradox (Cassirer 1902 [5, p. 187ff]). For a contemporary survey of the debate concerning this issue of Leibnizian scholarship the reader may consult (Sherry and Katz 2013 [64]).

Cassirer himself contended that the doctrines of the Marburg school, in particular his account of the role of idealizations in science and mathematics, might help to overcome the remaining obscurities that still beset Leibniz's account. According to him, for this endeavor it was essential to properly understand Leibniz' concept of motion that underlied his dynamical conception of geometry. From the viewpoint of classical Euclidean geometry, one may suspect that introducing the concept of motion into geometry amounts to an illicit confusion of pure geometry and empirical science. Cassirer vigorously argued against this interpretation:

It is not a systematical infringement to integrate the concept of motion into geometry. The concept that is dealt with here, is not from physics, but from logic: It denotes the conceptual continuation of the "principle" that was expressed in the concept of continuation. Thereby the concept of motion is separated from its empirical context and allocated in the area of pure and eternal "forms". ... The general achievement of the concept of motion resides in the formulation of the thought that the extensional being has to be constituted from an original lawful determination that preceeds it as its logical prius 20 (Cassirer 1902 [5, pp. 156-157])

In other words, motion in the realm of science is always lawful motion. The purely conceptual character of the concept of motion, its non-extensionality is shown by the concept of the differential (ibid. 157). This is not to say that Cassirer was not aware of the existence

\footnotetext{
${ }^{20} \mathrm{~A}$ prius is something that comes before or preceeds something else in some respect. The term was used in the philosophical jargon of the 19th century. Today it seems to be an outmoded Latinism.
} 
of non-continuous and perhaps even continuous but non-differentiable functions which had been vigorously discussed among mathematicians since Cauchy in the 1820s (cf. Hankel 1882 32]). Indeed, he explicitly pointed out that a more general notion of a function à la Dirichlet, perfectly made sense from a purely mathematical point of view ${ }^{21} \mathrm{He}$ only objected that such general functions were not meaningful for the determination of real processes of nature (cf. 217). He went on to declare that the meaningfulness of the limiting processes of the calculus "demonstrated" that the concept of (lawful) motion in nature was a non-empirical, logical notion:

If the transition to the quantitative zero does not eliminate the lawful character of the magnitude this is evidence that it (i.e., lawfulness) is not grounded in a quantitative principle. The magnitude must first disappear from our sensual perception before we can recognize its determinateness in the pure concept (Cassirer 1902, 157).

Here Cassirer does not distinguish between continuity and differentiability. As we shall see in a moment, this conflation enabled him to combine Cohen's "infinitesimal-centered" account with his own "functionoriented" one in an elegant but somewhat dubious way.

For the contemporary reader this passage may sound opaque, to put it mildly. We propose the following interpretation. Scientifically meaningful magnitudes obey continuous motions, i.e., motions the law of which could be described by a differentiable function $f$. In calculating the derivative of $f$, expressions such as $\lim \frac{f(x)-f\left(x^{\prime}\right)}{x-x^{\prime}}$ occur. These contain "quantitative zeroes" (if $x^{\prime}$ approximated $x$ ). This means that such expressions have no direct quantitative meaning. In particular they could not be perceived or experienced in any reasonable way. Nevertheless, conceptually, the calculation of the derivative makes perfect sense. Hence, the magnitude could be recognized as a meaningful and determinate magnitude, only after it had been submitted to a conceptual process (derivation) that eliminated all its sensual qualities. Continuity was thereby asserted to be a necessary presupposition for the constitution of nature as a possible object of rational investigation:

Continuity is a necessary presupposition for the existence of a mutually 1-1 relation between two series of change (Veränderungsreihen). This strand of thought is first formulated in Leibniz' best known formulation of the principle of continuity: "Datis ordinatis etiam quaesita sunt

\footnotetext{
${ }^{21}$ Compare footnote 8 .
} 
ordinata". The "data" denote the hypothetical conditions from which we start; the "quaesita" are the series of the conditioned that we look for. The order is thought as a law that determines the transition inside the two series in a continuous fashion. (ibid., 211 - 212)

Cassirer went on to contend that the standard "epsilon-delta" definition of continuity was merely a mild reformulation of Leibniz' original characterization of continuity "Datis ordinatis etiam quaesita sunt ordinata" (cf. ibid., 215). Thereby he could conclude that there was an intimate relation between Leibniz' continuity principle, the modern epsilon-delta definition of continuity, and Cohen's infinitesimalcentered account. Modern mathematics has shown that the relation is more complicated than Cassirer might have thought.

Nevertheless, despite its allegedly close relation to the modern mathematical concepts, according to Cassirer, Leibniz' continuity principle should not be understood as a mere mathematical definition. Here Cassirer is treading on somewhat dangerous ground. Leibniz's law of continuity had several meanings. It was not a single concept but rather a family of concepts; see for example (Jorgensen 2009 [36]). The problem is not merely the fact that the mathematical concept of continuity is not general enough to encompass Leibniz's concept. Rather, the law of continuity invokes several related concepts in a chain where the concepts at the extrema may be unrelated to each other at all. Perhaps one may say that Leibniz used this concept as an 'operative' one (see Subsection 2.2).

It would be a misunderstanding to read it simply as the claim that the processes of nature should be viewed as ready-made entities that were to be described in terms of continuous (or differentiable) functions:

The requirement of conceiving of nature ultimately as a complex of continuous functions (Inbegriff stetiger Funktionen) would not make sense, if the task of knowledge would be to reproduce a ready-made material descriptively. Continuity obtains its meaning only if it is conceived of as a basic act of the mind through which the subject conditions the object. (ibid., 218)

The principle of continuity should be understood as a guiding maxim for the evolution of scientific concepts that urges us to seek ever more profound systematic connections among them. Concepts should be connected in a uniform conceptual system and each concept should be transformable into each other continuously. For the Marburg school Leibniz' principle of continuity was the philosophical expression of one 
of the basic moments of modern science. It asserted that the welldefined and determinate character of scientific concepts did not reside in their isolation but in the lawfulness of their transitions (cf. (ibid. 201)).

Although Cassirer, faithfully following in the footsteps of his master Cohen, hailed Leibniz as the genius who made explicit for the first time the principle of continuity as a fundamental principle of modern science, already in Leibniz' System his assessment of Leibniz's achievements went in directions other than that of Cohen. The emerging differences between Cohen and Cassirer concerned the relation between the concepts of the infinitesimal and function. While Cohen emphasized that the "infinitesimal calculus (of Leibniz) had placed the concept of function, conceived of as a law of interdependency between two variable magnitudes, in the center of the methodology of mathematics" (Cohen 1902 [14, p. 239]), Cassirer put less emphasis on the role of the infinitesimal as a conceptual base for the concept of function. He generally praised Leibniz as an early partisan of a "functional" or "relational" worldview without mentioning infinitesimals at all:

If one understands by "substantial" worldview the conception according to which all beings and occurrences can be traced back to ultimate, rigid, absolute "things", then Leibniz' philosophy is strictly opposed to this standpoint. The tendency of Leibniz' philosophy that from now on will prevail in the ongoing progress of idealism points at a replacement of the older concept of being by the concept of function. (ibid., 486) [emphasis added-the authors]

In the evolution of Cassirer's own thought, this functional interpretation of the principle of continuity gained ever greater momentum and superseded the infinitesimal interpretation eventually leading to certain discrepancies with Cohen that surfaced in Cohen's letter to Cassirer dating august 24, 1910 (see Section 5).

4.4. Gawronsky's The Judgment of Reality. The second sustained effort to make sense of Cohen's approach is due to Dimitry Gawronsky (1883 - 1955). His dissertation under Cohen and Natorp was entitled Das Urteil der Realität (Gawronsky 1910 [24]). Although he was a close friend of Cassirer's, in the emerging discrepancies between Cohen and Cassirer on the relation between Cohen's 'infinitesimal' and Cassirer's 'functional' approach, he sought to find a mediating position between the two but eventually sided rather with Cohen than Cassirer. For this issue, two works of Gawronsky are relevant. Besides his already mentioned dissertation Das Urteil der Realität (henceforth 
Urteil), we have also his contribution Das Kontinuitätsprinzip bei Poncelet (Gawronsky 1912 [25]) to a Festschrift dedicated to Cohen on the occasion of his 70th anniversary in 1912. Today, Gawronsky's philosophical work has fallen into almost complete oblivion. Yet he was an important figure in the internal debate that took place within the Marburg school on matters infinitesimal between Cassirer, Natorp, and Cohen in the early years of the 20th century.

Gawronsky took upon himself the difficult task of updating Cohen's infinitesimal account, defending it against the less than orthodox accounts of Cassirer and Natorp. Probably his best known work among Cassirer scholars is the biographical article Ernst Cassirer: His Life and His Work (Gawronsky 1949 [26]) that appeared as a contribution to the Schilpp volume dedicated to Cassirer. As far as we know, the only contemporary discussion of Gawronsky's work and his role as a vigorous (although not uncritical) defender of Cohen's position against Cassirer (and, to a lesser extent, Natorp) is Massimo Ferrari's paper Dimitry Gawronsky and Ernst Cassirer: On the History of the Marburg School between Germany and Russia (Ferrari 2010 [20]) published in Russian.

In contrast to Cohen, Gawronsky was fully competent in matters of contemporary mathematics. He discussed the achievements of Bolzano, Grassmann, Cantor, Weierstrass, Veronese, and Dedekind with evident expertise. Moreover, Gawronsky expressed a positive appreciation of the limit method (cf. Ferrari [35, p. 249]).

Nevertheless, in contrast to Cassirer, Gawronsky sought to leave the philosophical core of Cohen's 'infinitesimal-centered' account intact. Hence, with respect to the infinitesimal approach he, rather than Cassirer or Natorp, may be considered as Cohen's true heir. His dissertation Das Urteil der Realität und seine mathematischen Voraussetzungen, literally 'The judgment of reality and its mathematical premises', may be regarded as the only serious attempt of amending Cohen's rather obscure pseudo-Kantian table of judgments.

In order to arrive at a better understanding of Gawronsky's Urteil, we have to recall that, as Gawronsky explains at the end of Urteil, the 'judgment of reality' refers to a distinction already made by Kant (and later modifed by Cohen) that can only be translated with difficulty into English. This is the distinction between Wirklichkeit and Realität that are both usually translated as reality. Roughly, reality in the sense of Realität is to mean 'the systematic knowledge of nature as it arises from the chaos of the immediately sensed' (unmittelbares Empfinden) (Gawronsky 1910 [24, p. 107]). Then the main thesis of Urteil is that 
the infinitesimal calculus plays a crucial role in the systematic knowledge of nature (or empirical reality) insofar as

the basic problem of objective empirical knowledge is the problem of change. But we only understand change if we obtain complete knowledge of the law that generates change, i.e., only if we can pursue the effect of the generating law in every infinitely small element of this change... And exactly this is achieved by the infinitesimal analysis. (Gawronsky 1910, [24, p. 105]).

In line with Cohen, Gawronsky asserted that "there is no other way to formulate and to justify the laws of nature than the infinitely small." (ibid.)

By identifying "reality" with the systematized knowledge of nature, Gawronsky saw "reality" as a "logical method" whose essence "was the assumption of the existence of a generating law". This "logical method" came along in two different ways, namely "by the method of number and by the method of the infinitesimal" (108). Both methods are carried out in the same three steps:

(1) Positing (Setzung)

(2) Infinite repetition (unendliche Wiederholung)

(3) Actual synthesis in a higher unity (aktuale Zusammenfassung in einer höheren Allheit)

Gawronsky's attempt to construe an analogy between the two methods is apparently based on the idea, which he shared with Dedekind and Cassirer, that the essence of numbers resided in their ordinal structure. More precisely, according to Gawronsky the conceptual generation of the natural numbers proceeded by first positing the unit ' 1 ' and then applying the generating principle of the successor function, thereby constituting the other natural numbers. This construction, however, had to be 'completed' by an "actual synthesis resulting in a higher unity". Or, formulated negatively, Gawronsky was not content with simply asserting that this repetition could go on and on leading to ever larger natural numbers. Rather, one had to look for a higher synthesis.

This was achieved, Gawronsky contended, by Cantor's theory of infinite ordinals. More precisely, Gawronsky conceived of Cantor's positing of the first infinite ordinal $\omega$ as the sought-for completion or synthesis. This completion of the natural numbers in terms of the first infinite ordinal $\omega$, however, was not simply the end of the constitution process of pure thought. On the contrary, it was just the beginning of a new stage in that it gave rise to a new infinite series generated by a new generating principle $\omega, \omega+1, \omega+2, \ldots$ This new series, 
then, had to be conceptually completed by positing $2 \omega$, which served as the starting point for a new series $2 \omega, 2 \omega+1,2 \omega+2, \ldots$ and so on. The determination of the limit of an infinite arithmetical series such as $3.1,3.14,3.141,3.1415, \ldots$ converging to $\pi$ had a similar conceptual structure, and even for the calculation of differentials and derivations Gawronsky assumed an analogous conceptual structure. According to him, they all followed the three-tiered pattern of 'positing', 'repetition', and 'actual synthesis'.

A modern mathematician may view Gawronsky's contention merely as the recognition that the construction of both the real numbers and infinitesimals involves infinitary constructions, a point made much later also by Robinson. But Gawronsky, following his master Cohen, made much more of it. According to him, the usage of infinitary constructions revealed the very essence of both (empirical) science and mathematics as being based on "pure thought". The transcendental analysis of science revealed that the origins of these methods were to be found in the 'judgment of reality'. Something that could not be counted 22 or differentiated, was not "real" in the sense that it could not possibly be the object of scientific knowledge 23 Differentiating and counting were the two basic methods of scientific knowledge. From a modern point of view, this may be a somewhat narrow and outdated characterization of the conceptual apparatus used in science and mathematics, but it certainly makes sense.

Let us now examine Das Kontinuitätsprinzip bei Poncelet (Gawronsky 1912). The main aim of this work was to elucidate Cohen's dictum that continuity is a basic law of thought (Denkgesetz) (Cohen 1902 [14, p. 76]). In Logik, Cohen traced the principle of continuity back to Leibniz. Furthermore, he offered the following high-sounding explication of the role of continuity in the ongoing process of philosophical and scientific thought:

Continuity is a law of thought. It is the law of thought of the connection which enables the generation of the unity of knowledge and thereby the unity of the object of knowledge. Continuity as a law of thought garantees the connection of all methods and disciplines of

\footnotetext{
${ }^{22}$ It should be noticed that Gawronsky here relied on a rather broad concept of counting that not only included "ordinary" counting but also various kinds of "infinite completion of counting".

${ }^{23}$ This has the apparently paradoxical consequence that there may be something real - in the sense of wirklich - that is not real. This apparent contradiction does not threaten in the original German and is avoided if one carefully distinguishes between the two meanings of "real" in Kant's language.
} 
mathematized empirical science (mathematische Naturwissenschaft). This law is therefore of crucial importance for the thinking of knowledge. Continuity is the law of knowledge. Continuity characterizes the basic feature of thought (Cohen 1902, 76, 77).

Gawronsky's aim in Das Kontinuitätsprinzip was to confirm and elucidate Cohen's global thesis on the central role of the principle of continuity for the evolution of scientific thought, by studying its role in the development of 19th century geometry exemplified in the work mainly of French geometers like Poncelet, Chasles, Carnot, and others. In line with Cohen, Gawronsky points out that this principle is not an achievement of 19th century science but a basic feature of all scientific thought. What is new, according to Gawronsky, is the way Poncelet applied the principle. The crucial point is not that new objects are subsumed under the known theorems and relations (cf. Gawronsky $1912,69)$ but that entire systems of theorems and relations themselves are 'continuously' modified and generalized (ibid. 71). This new interpretation of the principle entails that it must not be understood as an argument that generates mathematically secure results but rather as a heuristic principle that helps one find novel concepts whose relevant connections have yet to be secured by other means:

We see that the formation of concepts that is determined and guided by the principle of continuity cannot be completely justified by that principle alone. Rather, a subsequent check has to be carried out in order to determine the value of every newly introduced concept. (Gawronsky 1912 [25, p. 73])

For Gawronsky, the anticipatory and heuristic character of the principle of continuity in mathematics, as it was employed by Poncelet and others, was essential for discovering its true logical base that comes to the fore when we compare it with its "prototype" as it appears in the realm of pure thought:

Since the discovery of the principle of continuity, Hermann Cohen in his Logik der reinen Erkenntnis was the first who sought not only to evaluate the achievements of this principle in a comprehensive way, but also to determine its systematic significance, to introduce it in the system of pure thought and to render precise its position. He was the one who recognized this priniciple as a rather 
general and basic method of scientific thought, identifying it as a law of thought of knowledge. (Gawronsky $1912,74)$

From the viewpoint of Cohen's neo-Kantian approach, it is then the task of philosophy of science to integrate the issue of a purely mathematical evaluation of the principle of continuity, as it was understood by Poncelet and his contemporaries, into the general agenda of the transcendental logic of science that treats the basic methods of scientific knowledge Überhaupt (ibid. 76). For this purpose the philosopher must not rely on idle metaphysical speculations but has to know how this principle is actually applied in scientific practice. As an example of how this may be achieved in the case of geometry, Gawronsky discusses in detail two principles that Poncelet introduced in modern geometry, namely, the principles of central projections and his "théorie des polaires reciproques" (ibid., 76ff). This leads him to the conclusion that also Klein's Erlanger Programm, which proposes to define the essential properties of geometrical objects as invariants of certain transformations groups, can be unterstood as a realization of the principle of continuity (78). In sum, one may contend that Gawronsky's Das Kontinuitätsprinzip bei Poncelet offers a knowledgeable and not implausible narrative of the development and significance of the principle of continuity in 19th century geometry. This can be taken as indirect evidence that Husserl overstated his case when he summarily dismissed Cohen's account of the principle of continuity as a basic law of thought as "profound nonsense". Admittedly, it often takes considerable effort to distill some meaning out of Cohen's obscure prose, but the attempt to rescue at least some parts of Cohen's transcendental logic as presented in Die Logik der reinen Erkenntniss cannot be bluntly dismissed in Husserl's fashion.

4.5. Natorp's The Logical Foundations of the Exact Sciences. In his Die logischen Grundlagen der exakten Wissenschaften (Natorp 1910 [53]) the second leader of the Marburg school, Cohen's friend and colleague Paul Natorp also sought to come to terms with the problem of infinitesimals. He sought to develop a philosophically founded synthesis of two antagonistic, or at least very different, mathematical programs for the foundations of analysis. These are, on the one hand, the CDW program, which in Natorp's day had nearly achieved the status of a ruling orthodoxy, and on the other, the maverick, "infinitesimal-friendly" 
program of the Italian mathematician Giuseppe Veronese, put forward in his Fondamenti di geometria (Veronese 1892) 24

Natorp's contribution to the Marburg neo-Kantian philosophy in general, and to mathematics in particular, has been usually neglected compared to the better known works of Cohen and Cassirer. This may be considered, historically speaking, as an injustice, in particular with regard to the issue of infinitesimals. In Natorp's Die logischen Grundlagen der exakten Wissenschaften (Natorp 1910) we find the most elaborate and most complete discussion of limits and infinitesimals that any neo-Kantian philosopher ever published 25

Indeed, Natorp dedicated two chapters (namely, chapter III, 98 - 159, and chapter IV, 160 - 224) of Logische Grundlagen to a detailed criticism of the accounts of the various concepts of number as put forward by Frege, Dedekind, Cantor, Weierstrass, Pasch, and Veronese. Furthermore, Natorp believed that the accounts of Cantor and Veronese are compatible, and viewed Veronese as the "most eminent successor of Cantor" [53, p. 171]. He appears to have held that the differences between them were only technical differences of no conceptual and philosophical relevance. This was certainly an error, as Cantor and Veronese were well aware of the fact that their accounts differed in essential ways. Cantor rejected Veronese's numbers. Veronese was more tolerant, seeing Cantor's transfinite numbers and Veronese's own transarchimedean 26 infinitely large numbers as two admissible, but nevertheless quite different types of mathematical entities.

An analysis of Veronese's account would go beyond the scope of the present text. What we wish to explore is Natorp's philosophical motivation that inspired him to engage in the risky endeavor of sketching an all-embracing panoramic view of the landscape of the various kinds of number: 27 and their calculi (cf. Natorp 53]).

\footnotetext{
${ }^{24}$ See P. Cantù 4 for an extensive bibliography on Veronese.

${ }^{25}$ Die Logischen Grundlagen has never been translated into English. Even in German-speaking philosophy Natorp always remained in the shadow of the more brilliant Cassirer.

${ }^{26}$ This somewhat unusual coinage was utilized by Laugwitz [41, p. 104] and Peiffer-Reuter [56, p. 124f.].

${ }^{27}$ In retrospect, one can assert that Natorp's all-embracing vision was not merely a philosopher's pipe dream. It gained some mathematical substance over the decades. Thus, Kanovei and Reeken proved that there is a certain (class-size) structure ${ }^{*} V$ which is $\kappa^{+}$-saturated for every cardinal $\kappa$, together with an elementary embedding of the ZFC set universe $V$ into ${ }^{*} V$ (Kanovei and Reeken 2004 37. Theorem 4.3.17, p. 151]). (Ehrlich 2012 [19]), working in Von Neumann-BernaysGödel set theory with Global Choice, showed that a maximal (class) hyperreal field is isomorphic to the maximal surreal field.
} 
In line with all neo-Kantians, Natorp contended that intuition alone could not provide a foundation for our knowledge about infinitesimals, limits, and continuity. As he unequivocally put it: "Intuition cannot be a foundation for continuity, neither for space nor for time" [53, chapter IV, §4, p. 175].

According to him, the only relevant factor was "pure thought", i.e. the "transcendental method" or "transcendental logic" (see Subsection 3.1). Indeed, Natorp's insistence on the crucial role of an unfettered investigation of infinitary objects in applying the "transcendental method" gave his interpretation of infinitesimals in particular and numbers in general its specific flavor:

Numbers must not have any other basis than the laws of pure thought. (Natorp [53], ch. IV. §4, p.176).

His insistence on the infinitary character of the never-ending road of the "transcendental method" led him to criticize attempts to base the concept of number on something "finite" that only at a later stage of the conceptual evolution was overcome in favor of something "infinite" 28 For him, from the very start, the concept of number was soaked with the infinite. Hence, for him, any attempt to conceive of the "finite" rational numbers as a more solid base for allegedly the more elusive real numbers was philosophically mistaken. He appears even to have blamed Dedekind for having succumbed to such a temptation to some extent.

Natorp also came to formulate a perceptive criticism of a reductive aspect of Dedekind's approach of introducing new numbers as "cuts" of the set of rational numbers (cf. Dedekind 1872). Natorp pointed out that Dedekind assumed without justification that every cut 29 corresponds to exactly one non-rational number, which according to Natorp was a petitio principii. Of course, two distinct non-rational numbers which correspond to one and the same cut, cannot differ by a finite number. However, they could still differ by an infinitesimal 30 Thus,

\footnotetext{
${ }^{28}$ Natorp's insistence on the thoroughly infinite character of numbers, and the resulting emphasis on infinitesimals, was noted not only by his fellow philosophers but also by philosophically inclined mathematicians, regardless of whether they belonged to the CDW camp or were sympathetic to infinitesimals; see Fraenkel [21, footnote on pp. 50-51] and Robinson [59, p. 278].

${ }^{29}$ We will ignore the technical issue of cuts defined by the rationals themselves.

${ }^{30}$ This is indeed a mathematically coherent possibility as is shown already by the Levi-Civita fields 43 . developed at about the same time. In fact, any proper ordered field extension of the reals will have this property (for example, the hyperreal numbers).
} 
Dedekind's axiom that every cut corresponds to a unique number is an assumption that can be challenged.

Indeed, Natorp was the only neo-Kantian philosopher who ever explicitly evoked a suspension of the Archimedean axiom as an essential ingredient for any infinitesimal account (cf. [53, p. 169ff]). He evoked the Archimedean principle in his criticism of Cantor's alleged "proof" of the non-existence of infinitesimals (ibid.) 31

Natorp further points out that already the classical founding fathers of the concept of the infinitesimal, namely, Leibniz and Newton were well aware of the special status of the infinitesimal, often referred to by means of the modifier "intensive". The latter term seems to have had a meaning close to "non-Archimedean" to Natorp:

Already Galileo speaks of infinita non quanta; Leibniz contends the infinitesimal as praeter extensionem, imo extensione prius, for Newton the infinitesimal "moments" are not quantitates finitae, but principia iamiam nascentia finitarum magnitudinum; and Kant explains the infinitesimal through the intensive magnitude that include the base (Grund) for the extensive magnitudes, but is itself not extensive ([53, p. 170]).

Natorp further criticized Dedekind's assumption that the totality of all cuts is 'given' somehow although the converging rational series are to be considered just as procedures that allow us to approximate the irrational limit numbers if they exist.

Natorp's criticism of the idea that the set of Dedekind cuts is "given" could appear to be related to a criticism often voiced at the time, and that can be expressed in modern mathematical terms as follows. To say that the set of cuts is "given" is to make certain foundational assumptions, such as the axiom of infinity, usually accompanied by the classical interpretation of the existence quantifier, typically involving the law of excluded middle. If so, Natorp's criticism of Dedekind seems to echo criticisms of mathematicians like Kronecker and Brouwer. This, however, would be a misinterpretation. Natorp was neither an intuitionist à la Brouwer nor a platonist realist. Rather, Natorp was a critical idealist. For him, the ideal was neither something given "out there" nor did he require that it could be intuited in some way or other. For

\footnotetext{
${ }^{31}$ Natorp claimed that Cantor's "proof" was flawed for rather trivial reasons. His remarks are, however, too sketchy to be properly evaluated. Cantor was a competent mathematician and although he did make some mistakes, they were rather subtle ones. On the other hand, Natorp's competence in matters mathematical was that of an educated layman. Cantor's errors are analyzed in detail by Ehrlich [18] and Moore [48; see also (Proietti 2008 [57]).
} 
him, as well as for Cassirer, the existence of the ideal resided only in its function. This led him to perceive similarities between Dedekind's approach and that of du Bois-Reymond:

Apparently this kind of argumentation [i.e., Dedekind'sthe authors] is based on a way of thought that is manifest in P. du Bois-Reymond's Allgemeine Funktionentheorie (General Theory of Functions). This author introduces infinity and continuity by nothing short of an assumption (which he himself calls "idealist" but which is actually "realistic" in the sense of medieval scholasticism) that can often be seen in arithmeticians: namely, that the objects of mathematics exist in-themselves, and that these objects may have properties which our - always finite human thinking cannot fully grasp. (Natorp 1910, 180)

Natorp insisted on the thesis that the existence of mathematical objects can reasonably only mean that they are based on the mathematical thought (ibid.). Something that escapes mathematical thought, does not exist mathematically. In other words, du Bois-Reymond's conception of the continuum as something "that cannot be thought" did not make sense 32

What cannot be justified by mathematical thought, must not be posited by mathematics" (ibid. 180).

Despite certain alleged shortcomings in Dedekind's cut approach, Natorp saw Dedekind as being on the right track. The merit of having revealed the true kernel of Dedekind's method, is ascribed by Natorp to Weierstrass, Cantor, Pasch, and Veronese. He sees the basic flaw in Dedekind in the fact that Dedekind started his construction with the "finite", fully understood rational numbers, whereas the irrational numbers were considered as something derived 33 According to him, Weierstrass and Cantor made the decisive conceptual step of taking the infinite convergent "series" itself (Cantor's Fundamentalreihe) as a proper mathematical object to be considered in its own right. Then there is no longer any reason to distinguish between a "series" and its limit (cf. Natorp 1910 [53, p. 182]).

\footnotetext{
${ }^{32}$ At about the same time, a similar, but more elaborate critique of du BoisReymond's "empirist-idealist dialectics" was put forward in (Cassirer 1910 [7. p. 122ff]).

${ }^{33}$ This criticism of Natorp's is related to Cassirer's insistence on the "ontological equality" for the new entities (in this case, numbers) being introduced.
} 


\section{From infinitesimals to FUnCTIONAL CONCEPTS}

Cassirer sought to imbed Cohen's infinitesimals in a larger framework built upon his functional approach (cf. Cassirer 1912 [8]), but faithfully followed the general doctrine of the Marburg school not to dismiss infinitesimals as inconsistent pseudo-concepts. Unlike Cohen, however, he was interested not so much in infinitesimals per se as in a more profound and more precise understanding of the conceptual evolution as it took place in the modern sciences, in particular in physics and mathematics. For this purpose, he was led to a substantial recasting of the neo-Kantian framework of philosophy of science and mathematics as it had been designed by Cohen in Prinzip and Die Logik der reinen Erkenntnis.

In a nutshell, this amounted to placing the concept of function or relation center stage, rather than that of the infinitesimal. This project of Cassirer's began with his dissertation Leibniz' System in seinen wissenschaftlichen Grundlagen (Cassirer 1902 [5]). The project was succinctly presented in Kant und die moderne Mathematik (Cassirer 1907 [6]), and eventually culminated in Substanzbegriff und Funktionsbegriff (Cassirer 1910) and Die Philosophie der symbolischen Formen III (Cassirer 1929 [9]).

Cohen was not entirely happy with this development as shown by his letter to Cassirer dating from August 24 of 1910. Here Cohen first heaps lavish praise on his most brilliant disciple:

I heartily congratulate you and our entire community on your new and great achievement [i.e. the publication of SF-the authors]. If I shall not be able to write the second part of my Logik, no harm will be done to our common cause 34

Cohen then continues with the second half of his comment:

Yet, after my first reading of your book I still cannot discard as wrong what I told you in Marburg: you put the center of gravity upon the concept of relation and you believe that you have accomplished with the help of this concept the idealization of all materiality. You let even slip the remark that [the concept of relation] is a category. (...) Yet it is a category only insofar as it is a function, and function unavoidably demands the infinitesimal element in which alone the root of the ideal reality can be found.

\footnotetext{
${ }^{34}$ Cohen never published a second part of Logik.
} 
Among the recent interpretations of the relationship between Cohen's infinitesimals and Cassirer's relational approach, one can find conflicting views, both of which take this letter as their main piece of evidence. In his 2003 article Hermann Cohen's Das Prinzip der Infinitesimalmethode, Ernst Cassirer, and the Politics of Science in Wilhelmine Germany, Moynahan [51] put forward the thesis that Cassirer's relational account in SF should be understood as a more or less straightforward clarification of Cohen's Prinzip. As evidence for his claim, he quotes Cohen's 1910 letter to Cassirer. However, Moynahan only quotes the first half. The second half of the letter, in which Cohen pointed out the profound differences between SF and Prinzip, is not reproduced by Moynahan; see [51, p. 40].

In a more subtle and indirect way than Moynahan, recently Seidengart (2012) also argued that Cohen and Cassirer essentially agreed on the primordial role of the concept of the infinitesimal for modern science and its proper philosophical understanding.

To bring home his point Seidengart first reminded the reader that for Cohen the concept of the infinitesimal had to occupy center stage in any logic of modern science deserving of its name, since "infinitesimal analysis was the legitimate device of the mathematical science of nature." (Cohen, 1902, p. 30). According to Cohen, Leibniz, as the inventor of the infinitesimal calculus, was the one who brought about a situation where "mathematics became the mathematics of mathematized science of nature" (Cohen 1914 [15, p. 22]). As Seidengart rightly observes,

... independently of Kant, it was Leibniz who led Cohen along the pathway of his "logic of origin" ("Logik des Ursprungs"), which ... is the logic of pure thought ("Logik des reinen Denkens"). (Seidengart 2012 [62, p. 131])

However, Cohen's assessment of Leibniz had not always been thus positive. Only for Cohen's later thought, from Logik (1902) onwards, did Leibniz's philosophy play a pre-eminent role. In contrast, for Cassirer, Leibniz had always been the philosophical hero from the start of his philosophical career, as is evidenced by his Leibniz' System. Eventually, however, Seidengart concludes,

... inspite of the many innovations he was able to derive from Leibniz's infinitesimal analysis, Cohen aligned in the end the interpretation that young Cassirer laid out in his Leibniz' System and in his Erkenntnisproblem, both of which were explicitely cited by the founder 
of the Marburg School in 1914 [i.e., in (Cohen 1914 [15, p. 24])-the authors].

Since Cohen (1914) was the last time that Cohen dealt with Leibniz's infinitesimal analysis and its philosophical implications, this would appear to suggest that Cohen and Cassirer agreed on matters Leibnizian from the beginning of the 20th century until 1914 and perhaps even later, until Cohen's death in 1918.

This, however, is not quite true as is already shown by Cohen's letter of August 1910 where Cohen complained that Cassirer in SF had deviated from the party line as he no longer recognized the primacy of the concept of the infinitesimal.

Seidengart does not take into account the 1910 letter, and concentrates on (Cohen 1914 [15]). However, a closer look at Cohen (1914) reveals that this discrepancy of 1910, had not disappeared in 1914. True enough, in 1914 Cohen praised Cassirer's Leibniz' System (1902) and his Erkenntnisproblem (1906) as congenial elaborations of his own account of Leibniz and the role of the infinitesimal. More telling, however, is the fact that in 1914 Cohen did not cite Cassirer's Substance and Function (Cassirer 1910)! This omission suggests that the differences of 1910 between the two philosophers had not been resolved in the meantime. Rather, Cohen implicitely recognized in 1914 that his interpretation of Leibniz and that of Cassirer essentially differed.

Pursuing the opposite path, Skidelsky in his recent book Ernst Cassirer. The Last Philosopher of Culture [65] seeks to emphasize the differences between Cassirer and Cohen, as well as the alleged obsoleteness of the latter's infinitesimal account. He too invokes the 1910 letter, but leaves out the sentence in which Cohen characterized SF as a possible substitute for the second part of his Logik (cf. [65, p. 64]). Skidelsky seeks to drive home his case against Cohen by contending that "Cohen's theory of infinitesimals is in fact mistaken even from a purely mathematical point of view, being based on an outmoded interpretation of calculus" ([65, p. 65]).

The crown witness to Skidelsky's sweeping claim is, predictably, Russell's The Principles of Mathematics. Describing an infinitesimalist approach to the calculus as "outmoded" amounts merely to toeing the line on the CDW approach to the formalisation of analysis. The alleged uniqueness of such an approach is being increasingly challenged in the current literature. See, e.g., (Błaszczyk et al. 1013 [3]); (Bair et al. 2013 [1]).

Cohen concluded his criticisms by urging Cassirer to "take these thoughts into intimate consideration in the new edition" (of SF), but 
such an edition never appeared. Hence, Cassirer had to find another opportunity where he could pay due respects to Cohen's philosophical interpretation of infinitesimals. He appears to have attempted to overcome the clash with Cohen on matters infinitesimal in the article Hermann Cohen und die Erneuerung der Kantischen Philosophie (Cassirer 1912 [8]), dedicated to Cohen on the occasion of his 70th birthday. In this paper he hailed Cohen as the innovator and true heir of Kant's philosophy who had brought to the fore the fundamental principle of mathematized natural science in terms of the infinitesimal:

Matter and movement, force and mass may be conceptualized in this respect as instruments of knowledge. The high point of this development is not, however, reached before we come back to the basic mathematical motif underlying all specific conceptual formations of the natural sciences. This motif presents itself to us in the conceptual methodology of the "infinitesimal". (Cassirer 1912 [8, p. 260])

This appears to be a stronger endorsement of the infinitesimal approach than it really was. One should note that Cassirer spoke of the "methodology of the infinitesimal" rather than the "infinitesimal" itself. The methodology of the infinitesimal is something more general than the infinitesimal itself, and Cassirer seemed to have been well aware of this. Indeed, he sought to employ this greater generality to bind Cohen's infinitesimal approach with his own "relational approach". Actually he did not go beyond what he had already offered in SF some years earlier, when he praised the infinitesimal calculus as the first and most important example of the many calculi developed in modern mathematics, e.g. Grassmann's Ausdehnungslehre, Hamilton's theory of quaternions, the projective calculus of distances, and many others (cf. SF, 95). From this general perspective, then, it is easy to see that all these calculi are "relational" or "functional" in a broad sense. Meanwhile, the only example of a calculus that Cohen ever mentioned was the infinitesimal calculus. Superficially, Cassirer's remarks may appear to be a reconciliation of the infinitesimal approach and the relational approach, but they fail to convince. For instance, Cohen explicitly asserted that he was not primarily interested in the infinitesimal calculus, but rather in the specific philosophical ramifications of the concept of the infinitesimal which for Cohen represented the triumph of pure thought (cf. [13], p. 32). Hence, a general comparison of the infinitesimal calculus with other calculi probably did not overly 
impress Cohen. Cassirer might have felt this inadequacy and offered a further argument:

Without [the mathematical leitmotif of the infinitesimal], it would not be possible even to characterize rigorously the concept of movement 35 as it is presupposed by the mathematized natural science, to say nothing of the task of fully comprehending the lawfulness of movements. Thereby the circle of the critical investigations is closing. Since without doubt, the concept of the infinitely small does not denote a "Being" that can be captured by the senses, but a peculiar way and basic direction of thought 36 but this basic direction is now revealed as the necessary presupposition of the scientific object itself. (Cassirer 1912 [8, p. 260-261] 37

In fact, Cassirer never elaborated on the connection between Cohen's "infinitesimal analysis" and his own "relational analysis". Neither in SF nor in PSF did the concept of the infinitesimal play as prominent a role as in Cohen's Prinzip or his Logik der reinen Erkenntnis. Rather, Cassirer used Cohen's "methodology of the infinitesimal" only as a launch pad to develop his own "methodology of the relational".

Even more revealing is the fact that in the posthumously published fourth volume of The Problem of Knowledge ([11]), written in the late thirties during his Swedish exile, he had completely abandoned the infinitesimal standpoint; Cohen is not even mentioned once.

Cassirer's attempts in SF and elsewhere to connect his relational account with the infinitesimal account of Cohen are to be judged as less than fully convincing. Partisans of the infinitesimal approach should not blame Cassirer for this shortcoming too harshly, however. In his day, the effectiveness of an infinitesimal approach compared with one based on epsilontics was not too compelling. Moreover, the conceptualization of infinitesimals as an idealizing completion was not sufficiently understood to undertake a reasonable comparison with other idealizing completions. The advent of the various versions of modern

\footnotetext{
${ }^{35}$ Strictly speaking Cassirer's claim is inaccurate. It is possible to get by in mathematics without infinitesimals, as CDW had impressively shown.

${ }^{36}$ This cryptic remark is meant to emphasize the non-empirical character of the infinitesimal. Unlike ideal realists as well as empiricists, the Marburg school held that the infinitesimal was not to be found "out there" in some empirical or ideal domain independent of the cognizing subject; rather the infinitesimal was a way of thinking or conceptualizing the world.

${ }^{37}$ Similar remarks appear already in (SF, 1910 (130ff), 1953 (99ff)).
} 
infinitesimal-enriched continua has changed the conceptual landscape dramatically.

\section{ACKNOWLEDGMENTS}

T. Mormann was partially funded by the research project FF201233550 of the Spanish government. M. Katz was partially funded by the Israel Science Foundation grant no. 1517/12. We are grateful to the anonymous referees for a number of helpful suggestions.

\section{REFERENCES}

[1] Bair, J.; Błaszczyk, P.; Ely, R.; Henry, V.; Kanovei, V.; Katz, K.; Katz, M.; Kutateladze, S.; McGaffey, T.; Schaps, D.; Sherry, D.; Shnider, S.: Is mathematical history written by the victors? Notices of the American Mathematical Society 60 (2013) no. 8, to appear.

[2] Berkeley, G.: The Analyst, a Discourse Addressed to an Infidel Mathematician, 1734.

[3] Błaszczyk, P.; Katz, M.; Sherry, D.: Ten misconceptions from the history of analysis and their debunking. Foundations of Science 18 (2013), no. 1, 43-74. See http://dx.doi.org/10.1007/s10699-012-9285-8 and http://arxiv.org/abs/1202.4153

[4] Cantù, P.: Le concept de l'espace chez Veronese. Une comparaison avec la conception de Helmholtz et Poincaré, Philosophia Scientiae, 13/2 (2009), 129-149.

[5] Cassirer, E.: Leibniz' System in seinen wissenschaftlichen Grundlagen, Marburg. Elwert. 1902.

[6] Cassirer, E.: Kant und die moderne Mathematik Kant-Studien 12 (1907), $1-44$.

[7] Cassirer, E.: Substanzbegriff und Funktionsbegriff. Untersuchungen über die Grundlagen der Erkenntniskritik. Berlin. Bruno Cassirer. 1910. Translated in Substance and Function and Einstein's Theory of Relativity, New York, Dover Publications, 1953. Originally published 1923 by The Open Court Publishing Company, Chicago and LaSalle.

[8] Cassirer, E.: Hermann Cohen und die Erneuerung der Kantischen Philosophie Kant-Studien 17 (1912), 252-273.

[9] Cassirer, E.: Philosophie der symbolischen Formen, 3 Bände, Berlin, Bruno Cassirer, 1923 - 1929. Translated as The Philosophy of Symbolic Forms, 1953 - 1957, 3 Volumes, Yale University Press, New Haven.

[10] Cassirer, E.: Hermann Cohen 1842 - 1918. Social Research 10 (1943), 219 232.

[11] Cassirer, E.: The Problem of Knowledge, vol. 4. Philosophy, Science, and History since Hegel. Yale University Press. 1950.

[12] Coffa, A.: The Semantic Tradition from Kant to Carnap: To the Vienna Station. Cambridge, The University of Cambridge Press. 1991.

[13] Cohen, H.: Das Prinzip der Infinitesimalmethode und seine Geschichte. 1883.

[14] Cohen, H.: Logik der reinen Erkenntniss. Berlin, Bruno Cassirer. 1902. 
[15] Cohen, H.: Cohen, H., Einleitung mit kritischem Nachtrag zur "Geschichte des Materialismus" von F. A. Lange, 1914. Reedited by H. Holzhey, Hildesheim, Olms, 1984.

[16] Dedekind, R.: Essays on the Theory of Numbers, Chicago, Open Court, 1901(1872, 1888).

[17] Ehrlich, P.: Hahn's Über die nichtarchimedischen Grössensysteme and the Development of the Modern Theory of Magnitudes and Numbers to Measure them, in J. Hintikka (ed.) Essays on the Development of the Foundations of Mathematics, Boston, Kluwer, 165-213.

[18] Ehrlich, P.: The Rise of Non-archimedean Mathematics and the Roots of a Misconception I: The Emergence of non-Archimedean Systems of Magnitudes.Archive for the History of the Exact Sciences 60 (2006), 1-121.

[19] Ehrlich, P.: The absolute arithmetic continuum and the unification of all numbers great and small. Bulletin of Symbolic Logic, 18 (2012), no. 1, 1-45.

[20] Ferrari, M.: Dimitry Gawronsky i Ernst Kassirer: iz istorii Marburgskoj shkoly, in: neo-Kantianstvo nemeckoe i russkoe: mezhdu teoriej poznanija i kritikoj kultury, edited by I.N. Griftsova and N.A. Dmitrieva, Moskva, Rossijskaja politicheskaja enciklopedija, 2010, S. 242-256. [In Russian]

[21] Fraenkel, A.: Axiomatische Begründung von Hensels p-adischen Zahlen. Journal für die reine und angewandte Mathematik (Crelle's Journal) 141 (1912), 43-76.

[22] Friedman, M.: Dynamics of reason. Stanford, CLSI Publications.

[23] Friedman, M.: A Parting of the Ways: Carnap, Cassirer, and Heidegger. Chicago and LaSalle, Open Court, 2000.

[24] Gawronsky, D.: 1910, Das Urteil der Realität und seine mathematischen Voraussetzungen. Inauguraldissertation, Marburg.

[25] Gawronsky, D.: 1912, Das Kontinuitätsprinzip bei Poncelet. In Philosophische Abhandlungen, Hermann Cohen zum 70. Geburtstag dargebracht, Berlin, Bruno Cassirer, 65 - 84, 1912.

[26] Gawronsky, D.: Ernst Cassirer: His Life and Work, in P.A. Schilpp (ed.) The Philosophy of Ernst Cassirer, Evanston, Illinois, The Library of Living Philosophers, 3 - 37, 1949.

[27] Gerhardt, C. I. (ed.): Leibnizens mathematische Schriften (Berlin and Halle: Eidmann, 1850-1863).

[28] Gillies, D.: The Fregean revolution in logic. In Revolutions in mathematics, 265-305, Oxford Sci. Publ., Oxford Univ. Press, New York, 1992.

[29] Grattan-Guinness, I.: Solving Wigner's mystery: the reasonable (though perhaps limited) effectiveness of mathematics in the natural sciences. Mathematical Intelligencer 30 (2008), no. 3, 7-17.

[30] Griftsova, I.N.; Dmitrieva, N.A. (Eds.): Neo-Kantianstvo nemeckoe i russkoe: mezhdu teoriej poznanija i kritikoj kultury (Russian) [Neo-Kantianism, German and Russian: between a theory of knowledge and critique of culture]. Moskva, Rossijskaja politicheskaja enciklopedija, 2010. [In Russian]

[31] Haaparanta, L.: The Relation between Logic and Philosophy, 1874-1931, in L. Haaparanta, The Development of Modern Logic (ed.), Oxford, Oxford University Press. 222-262, 2009.

[32] Hankel, H.: "Untersuchungen über die unendlich oft oszillierenden und unstetigen Funktionen. Mathematische Annalen 20 (1882) no. 1, 63-112. 
[33] Heis, J.: "Critical philosophy begins at the very point where logistic leaves off": Cassirer's response to Frege and Russell. Perspectives on Science $\mathbf{1 8}$ (2010), no. 4, 383-408.

[34] Heis, J.: Ernst Cassirer's Neo-Kantian philosophy of geometry. British Journal of History of Philosophy 19 (2011), no. 4, 759-794.

[35] Hewitt, E.: Rings of real-valued continuous functions. I. Transactions of the American Mathematical Society 64 (1948), 45-99.

[36] Jorgensen, L.: The Principle of Continuity and Leibniz's Theory of Consciousness. Journal of the History of Philosophy 47 (2009), no. 2, 223-48.

[37] Kanovei, V.; Reeken, M.: Nonstandard analysis, axiomatically. Springer Monographs in Mathematics, Berlin: Springer, 2004.

[38] Katz, M.; Sherry, D.: Leibniz's laws of continuity and homogeneity. Notices of the American Mathematical Society 59 (2012), no. 11, 1550-1558. See http://www.ams.org/notices/201211/and http://arxiv.org/abs/1211.7188

[39] Katz, M.; Sherry, D.: Leibniz's infinitesimals: Their fictionality, their modern implementations, and their foes from Berkeley to Russell and beyond. Erkenntnis (2013) (online first), see http://dx.doi.org/10.1007/s10670-012-9370-y and http://arxiv.org/abs/1205.0174

[40] Kühn, M.: Interpreting Kant Correctly: On the Kant of the Neo-Kantians, in R.A. Makkreel, S. Luft (eds.) Neo-Kantianism in Contemporary Philosophy, Bloomington and Indianopolis, Indiana University Press, 113 - 131, 2010.

[41] Laugwitz, D.: Debates about infinity in mathematics around 1890: the Cantor-Veronese controversy, its origins and its outcome. NTM (N.S.) (NTM Neue Serie. Internationale Zeitschrift für Geschichte und Ethik der Naturwissenschaften, Technik und Medizin. New Series. International Journal of History and Ethics of Natural Sciences, Technology and Medicine) 10 (2002), no. $2,102-126$.

[42] Leibniz, G.: (1702) Letter to Varignon, 2 feb. 1702, in Gerhardt 27, vol. IV, pp. 91-95].

[43] Levi-Civita, T.: Sugli infiniti ed infinitesimi attuali quali elementi analitici, Atti del R. Istituto Veneto di Scienze Lettre ed Arti, Venezia (Serie 7) 4 (1892-93), 1765-1815. Reprinted in [44.

[44] Levi-Civita, T.: Tullio Levi-Civita, Opere Matematiche, Memorie e Note, Volume primo 1893-1900, Nicola Zanichelli, Bologna, 1954.

[45] Liebmann, O.: 1865 (1912), Kant und die Epigonen. Eine kritische Abhandlung. Berlin, Verlag von Reuther und Reichard.

[46] McCarty, D.C.: Problems and Riddles: Hilbert and the Du Bois-Reymonds. Synthese 147 (2005), no. 1, 63-79.

[47] Mohanty, J.N.: (2008), The Philosophy of Edmund Husserl. A Historical Development, New Haven and London, Yale University Press.

[48] Moore, M.E.: A Cantorian Argument Against Infinitesimals. Synthese 133 (2002), 305-330.

[49] Mormann, T.: Mathematical Metaphors in Natorp's Neokantian Epistemology and Philosophy of Science, in M.H.G. Hoffmann, J. Lenhard, F. Seeger (eds.), Activity and Sign - Grounding Mathematics Education, Festschrift for M. Otte, Berlin and New York, Springer, 2005, 229-239.

[50] Mormann, T.: Idealization in Cassirer's philosophy of mathematics. Philosophia Mathematica (3) 16 (2008), no. 2, 151-181. 
[51] Moynahan, G.B.: Hermann Cohen's Das Prinzip der Infinitesimalmethode, Ernst Cassirer, and the Politics of Science in Wilhelmine Germany. Perspectives on Science 11 (2003), No. 1, 35-75.

[52] Natorp. P.: 1903, Philosophische Propädeutik (Allgemeine Einleitung in die Philosophie und Anfangsgründe der Logik, Ethik und Psychologie), Marburg, Elwert. 68 pages.

[53] Natorp, P.: 1910 (1921), Die logischen Grundlagen der exakten Wissenschaften, Leipzig und Berlin, Teubner.

[54] Natorp, P.: 1912, Kant und die Marburger Schule. Kant-Studien 17 (1912), 193-206.

[55] Orth, E. W.: 1996, Von der Erkenntnistheorie zur Kulturphilosophie, Würzburg, Königshausen und Neumann.

[56] Peiffer-Reuter, R.: L'infini relatif chez Veronese et Natorp. Un chapitre de la prehistoire de l'analyse non-standard, en H. Barreau, J. Harthong (Eds.), 1989, La mathématique non-standard, 117 - 142. Paris. Editions du CNRS.

[57] Proietti, C.: Natural Numbers and Infinitesimals: A Discussion between Benno Kerry and Georg Cantor. History and Philosophy of Logic 29 (2008), no. 4, 343-359.

[58] Robinson, A.: Non-standard analysis. Nederl. Akad. Wetensch. Proc. Ser. A $\mathbf{6 4}=$ Indag. Math. 23 (1961), 432-440 [reprinted in Selected Works, see item [60, pp. 3-11]

[59] Robinson, A.: Non-standard analysis. North-Holland Publishing Co., Amsterdam 1966.

[60] Robinson, A.: Selected papers of Abraham Robinson. Vol. II. Nonstandard analysis and philosophy. Edited and with introductions by W. A. J. Luxemburg and S. Körner. Yale University Press, New Haven, Conn., 1979.

[61] Russell, B.: The Principles of Mathematics. Routledge. London 1903.

[62] Seidengart, J.: Cassirer, Reader, Publisher, and Interpreter of Leibniz's Philosophy. R. Krómer and Y. Chin-Drian (eds.), New Essays in Leibniz Reception: In Science and Philosophy of Science 1800-2000, Springer, Basel, 2012.

[63] Sherry, D.: The wake of Berkeley's Analyst: rigor mathematicae? Stud. Hist. Philos. Sci. 18 (1987), no. 4, 455-480.

[64] Sherry, D.; Katz, M.: Infinitesimals, imaginaries, ideals, and fictions. Studia Leibnitiana, to appear.

[65] Skidelsky, E.: Ernst Cassirer. The Last Philosopher of Culture, Princeton NJ, Princeton University Press, 2008.

[66] Weyl, H.: Die heutige Erkenntnislage in der Mathematik, Symposion 1, 1-32, 1925. Reprinted in H. Weyl, 1968, Gesammelte Abhandlungen, vol. 2, Berlin, Springer. English Translation in P. Mancosu (ed.) From Brouwer to Hilbert. The Debate on the Foundations of Mathematics in the 1920s, Oxford, Oxford University Press.

[67] Weyl, H.: The Future of Mathematics. The American Mathematical Monthly 58 (1951), no. 8, 523-553.

[68] Wigner, E.: The unreasonable effectiveness of mathematics in the natural sciences [Comm. Pure Appl. Math. 13 (1960), 1-14]. Mathematical analysis of physical systems, 1-14, Van Nostrand Reinhold, New York, 1985. 
T. Mormann, Department of Logic and Philosophy of Science, University of the Basque Country UPV/EHU, 20080 Donostia San SebasTIAN, SPAIN

E-mail address: ylxmomot@sf.ehu.es

M. Katz, Department of Mathematics, Bar Ilan University, Ramat GAN 52900 ISRAEL

E-mail address: katzmik@macs.biu.ac.il 